\title{
Super Toeplitz Operators and Non-Perturbative Deformation Quantization of Supermanifolds
}

\author{
David Borthwick $^{1}$, Slawomir Klimek ${ }^{2}$, Andrzej Lesniewski ${ }^{1 \star}$, and \\ Maurizio Rinaldi ${ }^{\star \star \star}$ \\ ${ }^{1}$ Lyman Laboratory of Physics, Harvard University, Cambridge, MA 02138, USA \\ 2 Department of Mathematics, IUPUI, Indianapolis, IN 46205, USA
}

Received April 7, 1992; in revised form August 3, 1992

\begin{abstract}
The purpose of this paper is to construct non-perturbative deformation quantizations of the algebras of smooth functions on Poisson supermanifolds. For the examples $U^{1 \mid 1}$ and $C^{m \mid n}$, algebras of super Toeplitz operators are defined with respect to certain Hilbert spaces of superholomorphic functions. Generators and relations for these algebras are given. The algebras can be thought of as algebras of "quantized functions," and deformation conditions are proven which demonstrate the recovery of the super Poisson structures in a semi-classical limit.
\end{abstract}

\section{Introduction}

I.A. Deformation quantization is a natural scheme for constructing non-commutative spaces, in the sense of [10], as deformations of Poisson manifolds. In this framework, the algebra of functions on a manifold is replaced by a family of non-commuting algebras of "quantized functions," which are indexed by a parameter ("Planck's constant"). The guiding principle of the deformation quantization construction is that the classical algebra of functions is obtained from the quantized algebras in the limit as Planck's constant goes to zero, with a first order correction determined by the Poisson structure on the manifold. This scheme was originally proposed in the context of a formal power series in the deformation parameter $[2,4]$, but has recently been extended to the non-perturbative setup (see $[18,19]$, and references therein).

This non-perturbative scheme was applied to the Poincaré disc in [12], with an approach using Toeplitz operators as quantization maps based on the ideas of [3-6] (Toeplitz operators were also used to quantize the sphere in [20]). The techniques of [12] have been applied to compact Riemann surfaces in [13], to the $n$-dimensional complex vector space in [9], to a two-parameter deformation of the unit disc [14], and to the four large classes of Cartan domains in [8]. The basic

\footnotetext{
* Supported in part by the Department of Energy under grant DE-FG02-88ER25065
}

$\star \star$ Supported in part by the Italian National Institute for Nuclear Physics (INFN) 
ingredients for the procedure were a group of automorphisms, a symplectic form invariant under the action of the group, and a perturbation of the invariant measure depending upon a parameter related to Planck's constant. The Toeplitz operators were defined with respect to the Hilbert spaces of holomorphic functions which were square integrable in the perturbed measures.

In this paper, we extend these techniques to the case of Poisson supermanifolds $[7,15,16]$. The basic concepts of Poisson supermanifolds and of our procedure are outlined below.

I.B. Let $\mathscr{M}=(M, \mathcal{O})$ be a smooth supermanifold (see e.g. $[7,15,17]$ ) of finite dimension. Here, $M$ is an ordinary smooth manifold, called the base of $\mathscr{M}$, and $\mathcal{O}$ is a sheaf of supercommutative superalgebras, called the sheaf of smooth functions on $\mathscr{M}$. Let $C^{\infty}(\mathscr{M})$ denote the superalgebra of global sections of $\mathcal{O}$. In this paper, we will be concerned with Poisson supermanifolds, i.e. supermanifolds for which $C^{\infty}(\mathscr{M})$ is a Poisson superalgebra $[7,16]$. This means that $C^{\infty}(\mathscr{M})$ is equipped with a bilinear mapping

$$
\{\cdot, \cdot\}: C^{\infty}(\mathscr{M}) \times C^{\infty}(\mathscr{M}) \rightarrow C^{\infty}(\mathscr{M})
$$

called a super Poisson bracket, which satisfies the conditions:

$$
\begin{gathered}
\{f, g\}=(-1)^{p(f) p(g)+1}\{g, f\} \\
(-1)^{p(f) p(h)}\{f,\{g, h\}\}+(-1)^{p(h) p(g)}\{h,\{f, g\}\}+(-1)^{p(g) p(f)}\{g,\{h, f\}\}=0, \\
\{f, g h\}=\{f, g\} h+(-1)^{p(f) p(g)} g\{f, h\}
\end{gathered}
$$

where $f, g, h \in C^{\infty}(\mathscr{M})$, and where $p(f) \in\{0,1\}$ is the parity of the (homogeneous) element $f \in C^{\infty}(\mathscr{M})$. Conditions (I.2) and (I.3) say that $C^{\infty}(\mathscr{M})$ is a Lie superalgebra, while condition (I.4) says that the super Poisson bracket obeys the super Leibniz rule. Poisson supermanifolds arise in physics as phase spaces for classical systems involving both bosons and fermions.

In the examples discussed in this paper, $\mathscr{M}$ is supersymplectic (in fact, super Kähler), i.e. it comes equipped with a supersymplectic (by which we mean even, closed and non-degenerate) two-form $\omega$. Furthermore, the superalgebra $C^{\infty}(\mathscr{M})$ has a natural *-structure. A supersymplectic form defines a super Poisson bracket, just as an ordinary symplectic form defines a Poisson bracket. This comes about as follows $[7,15]$. In local coordinates,

$$
\omega=\sum_{j, k} d X_{k} \wedge d X_{j} \omega_{j k}
$$

where the supermatrix $\left\{\omega_{j k}\right\}$ satisfies

$$
\omega_{j k}=(-1)^{\left(\varepsilon_{j}+1\right)\left(\varepsilon_{k}+1\right)} \omega_{k j}
$$

with $\varepsilon_{j}:=p\left(X_{j}\right)$. Let $\left\{\omega^{j k}\right\}$ be the inverse of $\left\{\omega_{j k}\right\}$, i.e. $\sum_{l} \omega^{j l} \omega_{l k}=\delta_{j k}$. Then

$$
\{f, g\}:=\sum_{j, k}(-1)^{\left(p(f)+\varepsilon_{j}\right) \varepsilon_{k}} \omega^{j k} \frac{\partial f}{\partial X_{j}} \frac{\partial g}{\partial X_{k}}
$$

is a super Poisson bracket. 
The examples studied in this paper are the super unit disc $U^{1 / 1}$ and the superspace $\mathbb{C}^{m \mid n}$. These supermanifolds and their supersymplectic structures are defined in Sects. II and VI, respectively.

I.C. The goal of this paper is to study, in a rigorous, non-perturbative way, quantizations of Poisson supermanifolds. We restrict attention to the two examples mentioned in Sect. I.B. We believe that the methods explained here can be generalized to include, for example, super Grassmannians and Cartan superdomains (for the bosonic case see [8] and references therein).

By a (deformation) quantization of a Poisson supermanifold $\mathscr{M}$, we refer to a family of normed $*$-superalgebras $\mathscr{A}_{\mu}(\mathscr{M})$, parametrized by a Planck's constant $\mu \in(0,1)$ (say), and a grading preserving $*$-homomorphism of vector superspaces $C^{\infty}(\mathscr{M}) \ni f \rightarrow T^{(\mu)}(f) \in \mathscr{A}_{\mu}(\mathscr{M})$ such that

$$
\lim _{\mu \rightarrow 0}\left\|\frac{1}{\mu}\left[T^{(\mu)}(f), T^{(\mu)}(g)\right]-T^{(\mu)}(\{f, g\})\right\|_{\mu}=0,
$$

where $\|\cdot\|_{\mu}$ is the norm on $\mathscr{A}_{\mu}(\mathscr{M})$. In the above formula and throughout this paper, $[\cdot, \cdot]$ denotes the graded commutator. This notion of quantization is closely related to that of $[18,19]$. We think of the elements of $\mathscr{A}_{\mu}(\mathscr{M})$ as "functions on the (quantized) non-commutative superspace." In our examples, $\mathscr{A}_{\mu}(\mathscr{M})$ generates a $\mathbb{C}^{*}$-algebra, and this could be made part of the definition (it is natural to do so in the purely bosonic case). It appears to us, however, that $\mathbb{C}^{*}$-algebras are rather unnatural in the super-context.

The central notion of our construction is that of a super Toeplitz operator, defined in Sects. III and VI. Super Toeplitz operators are super-analogs of the usual (bosonic) Toeplitz operators (see e.g. $[1,21]$ and references therein) and share some of their properties. They are defined on certain $\mathbb{Z}_{2}$-graded Hilbert spaces of superholomorphic functions on $\mathscr{M}$. The quantization map $C^{\infty}(\mathscr{M}) \ni f \rightarrow T^{(\mu)}(f)$ $\in \mathscr{A}_{\mu}(\mathscr{M})$ introduced above is just a map assigning to a symbol the corresponding super Toeplitz operator, and $\mathscr{A}_{\mu}(\mathscr{M})$ is the $*$-algebra generated by super Toeplitz operators with smooth bounded symbols.

I.D. The paper is organized as follows. In Sect. II, we study the properties of the super unit disc $U^{1 / 1}$. In Sect. III, we construct a quantization of $U^{1 / 1}$ by means of super Toeplitz operators. The structure of the resulting $\mathbb{C}^{*}$-algebra of "quantized continuous functions" is studied in Sect. IV. Section V contains the proof of the deformation estimate (I.8). In Sects. VI and VII, we construct the quantization for the superspace $\mathbb{C}^{m \mid n}$.

\section{The Supermanifold $U^{1 \mid 1}$}

II.A. In this section we describe the simplest hyperbolic supermanifold, namely the super unit disc. We will discuss the quantization of this space in the next section. The super unit disc $\mathscr{U} \equiv U^{1 \mid 1}$ is the supermanifold $(U, \mathcal{O})$, where $U=\{z \in \mathbb{C}:|z|<1\}$ is the open unit disc, and where $\mathcal{O}$ is the sheaf of superalgebras on $U$ whose space of global sections is $C^{\infty}(\mathscr{U}):=C^{\infty}(U) \otimes \bigwedge(\mathbb{C})$, where $\bigwedge(\mathbb{C})$ denotes the exterior algebra over $\mathbb{C} \cong \mathbb{R}^{2}$. In the following, we will refer to the 
elements of $C^{\infty}(\mathscr{U})$ as smooth functions on $\mathscr{U}$. We denote the standard generators of $\bigwedge(\mathbb{C})$ by $\theta$ and $\bar{\theta}$. An element $f \in C^{\infty}(\mathscr{U})$ can thus be written as

$$
f(z, \theta, \bar{\theta})=f_{00}(z)+f_{10}(z) \theta+f_{01}(z) \bar{\theta}+f_{11}(z) \theta \bar{\theta},
$$

where $f_{j k} \in C^{\infty}(U)$.

A function $f \in C^{\infty}(\mathscr{U})$ is called bounded if all the components $f_{j k}$ and their derivatives are bounded functions on $U$. We let $C_{b}^{\infty}(\mathscr{U})$ denote the superspace of bounded functions on $\mathscr{U}$. We give $C_{b}^{\infty}(\mathscr{U})$ the topology of a Frechet space. This topology is defined by the family of norms:

$$
\|f\|_{t}:=\sum_{m+n \leqq t} \sum_{0 \leqq j, k \leqq 1} \sup _{|z| \leqq 1}\left|\partial_{z}^{m} \partial_{\bar{z}}^{n} f_{j k}(z)\right|,
$$

where $t \geqq 0$.

A function $f \in C^{\infty}(\mathscr{U})$ is called superholomorphic, if $\partial_{\bar{z}} f=\partial_{\bar{\theta}} f=0$ or, equivalently, if

$$
f(z, \theta)=f_{0}(z)+f_{1}(z) \theta,
$$

with $f_{0}$ and $f_{1}$ holomorphic. We will find it convenient to use a collective notation for the generators of $C^{\infty}(\mathscr{U})$, namely $Z:=(z, \theta)$.

II.B. The super unit disc $\mathscr{U}$ admits an action of the Lie supergroup $S U(1,1 \mid 1)$. Recall that this supergroup is defined as follows. Its base manifold is $S U(1,1)$, and its structure sheaf is generated by $\gamma_{j k}$ and $\bar{\gamma}_{j k}, 1 \leqq j, k \leqq 3$, with the parity assignments:

$$
p\left(\gamma_{j k}\right)=p\left(\bar{\gamma}_{j k}\right)= \begin{cases}0, & \text { if } 1 \leqq j, k \leqq 2 \text { or } j=k=3, \\ 1, & \text { otherwise } .\end{cases}
$$

Let $\gamma=\left\{\gamma_{j k}\right\}$ denote the supermatrix with entries $\gamma_{j k}$ and let $\gamma^{*}$ denote its hermitian adjoint, $\gamma_{j k}^{*}:=\bar{\gamma}_{k j}$. We require that

$$
\gamma^{*} J \gamma=J
$$

where

$$
J=\left(\begin{array}{rrr}
1 & 0 & 0 \\
0 & -1 & 0 \\
0 & 0 & -1
\end{array}\right),
$$

and that

$$
\operatorname{Ber} \gamma=1,
$$

where Ber denotes the Berezinian. Recall that the Berezinian of a block supermatrix

$$
M=\left(\begin{array}{ll}
A & B \\
C & D
\end{array}\right),
$$

with $A, D$ even, $D$ invertible, and $B, C$ odd, is defined by

$$
\operatorname{Ber} M:=\operatorname{det}\left(A-B D^{-1} C\right) \operatorname{det} D^{-1} \text {. }
$$


Conditions (II.5) and (II.7) are the relations defining the structure sheaf of $S U(1,1 \mid 1)$. The multiplication on $S U(1,1 \mid 1)$ is defined in the obvious way. In the following, we will refer to the supermatrices of generators of $S U(1,1 \mid 1)$ as the elements of $S U(1,1 \mid 1)$. A simple calculation shows that the formulas below define an action of $S U(1,1 \mid 1)$ on $\mathscr{U}$ :

$$
\begin{aligned}
& z \rightarrow z^{\prime}:=\frac{\gamma_{11} z+\gamma_{12}+\gamma_{13} \theta}{\gamma_{21} z+\gamma_{22}+\gamma_{23} \theta}, \\
& \theta \rightarrow \theta^{\prime}:=\frac{\gamma_{31} z+\gamma_{32}+\gamma_{33} \theta}{\gamma_{21} z+\gamma_{22}+\gamma_{23} \theta} .
\end{aligned}
$$

The expression $\left(\gamma_{21} z+\gamma_{22}+\gamma_{23} \theta\right)^{-1}$ is defined in terms of the standard Taylor series for superfunctions [7]. By a slight abuse of notation, we write (II.10) as $Z^{\prime}=\gamma(Z)$.

Define

$$
\gamma^{\prime}(Z)=\operatorname{Ber}\left(\begin{array}{ll}
\frac{\partial z^{\prime}}{\partial z} & \frac{\partial \theta^{\prime}}{\partial z} \\
\frac{\partial z^{\prime}}{\partial \theta} & \frac{\partial \theta^{\prime}}{\partial \theta}
\end{array}\right) \equiv \operatorname{Ber} \frac{\partial Z^{\prime}}{\partial Z}
$$

Proposition II.1. If $\gamma \in S U(1,1 \mid 1)$, then

$$
\gamma^{\prime}(Z)=\frac{1}{\gamma_{21} z+\gamma_{22}+\gamma_{23} \theta} .
$$

Proof. The proof is by explicit computation. The supermatrix of derivatives, written in the notation of (II.11), is the following,

$$
\frac{\partial Z^{\prime}}{\partial Z}=\frac{1}{\gamma_{21} z+\gamma_{22}+\gamma_{23} \theta}\left(\begin{array}{ll}
\gamma_{11}-\gamma_{21} z^{\prime} & \gamma_{31}-\gamma_{21} \theta^{\prime} \\
\gamma_{13}-\gamma_{23} z^{\prime} & \gamma_{33}+\gamma_{23} \theta^{\prime}
\end{array}\right) \text {. }
$$

Taking the Berezinian of this supermatrix, we have

$$
\gamma^{\prime}(Z)=\left[\gamma_{11}-\gamma_{21} z^{\prime}-\frac{\left(\gamma_{31}-\gamma_{21} \theta^{\prime}\right)\left(\gamma_{13}-\gamma_{23} z^{\prime}\right)}{\gamma_{33}+\gamma_{23} \theta^{\prime}}\right] \frac{1}{\gamma_{33}+\gamma_{23} \theta^{\prime}} .
$$

We substitute the expressions for $z^{\prime}$ and $\theta^{\prime}$, and with a little manipulation we obtain

$$
\gamma^{\prime}(Z)=\frac{\operatorname{Ber} \gamma}{\gamma_{21} z+\gamma_{22}+\gamma_{23} \theta}
$$

We set

$$
Z \bar{W}:=z \bar{w}+\theta \bar{\eta},
$$

where $Z=(z, \theta), W=(w, \eta)$, and consider the expression $1-Z \bar{W}$.

Proposition II.2. With the above definitions,

$$
1-\gamma(Z) \overline{\gamma(W)}=(1-Z \bar{W}) \gamma^{\prime}(Z) \overline{\gamma^{\prime}(W)} .
$$

Proof. The identity follows immediately from the requirement (II.5) and from Proposition II.1. 
II.C. The superalgebra $C^{\infty}(\mathscr{U})$ can be equipped with an $S U(1,1 \mid 1)$-invariant super Poisson structure. This arises as follows. Let $\Omega^{1}(\mathscr{U})$ and $\Omega^{-1}(\mathscr{U})$ denote the $C^{\infty}(\mathscr{U})$-modules of 1 -forms and vector fields on $\mathscr{U}$, respectively. The following elements of $\Omega^{1}(\mathscr{U}) \otimes \Omega^{-1}(\mathscr{U})$,

$$
\begin{aligned}
& Q:=\sum_{0 \leqq j \leqq 1} d Z_{j} \otimes \frac{\partial}{\partial Z_{j}}, \\
& \bar{Q}:=\sum_{0 \leqq j \leqq 1} d \bar{Z}_{j} \otimes \frac{\partial}{\partial \bar{Z}_{j}},
\end{aligned}
$$

are clearly $S U(1,1 \mid 1)$-invariant. Consider the following two-form,

$$
\begin{aligned}
\omega & :=Q \bar{Q} \log (1-Z \bar{Z}) \\
& =\sum_{j, k}(-1)^{p\left(\bar{Z}_{k}\right)+1} d \bar{Z}_{k} \wedge d Z_{j} \frac{\partial^{2}}{\partial Z_{j} \partial \bar{Z}_{k}} \log (1-Z \bar{Z}) .
\end{aligned}
$$

Proposition II.3. $\omega$ is an $S U(1,1 \mid 1)$-invariant supersymplectic form on $\mathscr{U}$.

Proof. To see that $\omega$ is $S U(1,1 \mid 1)$-invariant, we note that, as a consequence of (II.17),

$$
\log (1-\gamma(Z) \overline{\gamma(Z)})=\log (1-Z \bar{Z})+\log \gamma^{\prime}(Z)+\log \overline{\gamma^{\prime}(Z)} .
$$

Since $\gamma^{\prime}(Z)$ is holomorphic,

$$
Q \bar{Q} \log \gamma^{\prime}(Z)=Q \bar{Q} \log \overline{\gamma^{\prime}(Z)}=0,
$$

and so $\gamma^{*} \omega=\omega$, as claimed.

To see that $\omega$ is supersymplectic, we write

$$
\omega=-d z \wedge d \bar{z} \omega_{z \bar{z}}+d z \wedge d \bar{\theta} \omega_{z \bar{\theta}}+d \theta \wedge d \bar{z} \omega_{\theta \bar{z}}+d \theta \wedge d \bar{\theta} \omega_{\theta \bar{\theta}},
$$

where

$$
\begin{aligned}
& \omega_{z \bar{z}}=\frac{1-\theta \bar{\theta}}{(1-z \bar{z}-\theta \bar{\theta})^{2}}=\frac{1}{(1-z \bar{z})^{2}}+\frac{1+z \bar{z}}{(1-z \bar{z})^{3}} \theta \bar{\theta}, \\
& \omega_{z \bar{\theta}}=\frac{\bar{z} \theta}{(1-z \bar{z}-\theta \bar{\theta})^{2}}=\frac{\bar{z} \theta}{(1-z \bar{z})^{2}}, \\
& \omega_{\theta \bar{z}}=\frac{z \bar{\theta}}{(1-z \bar{z}-\theta \bar{\theta})^{2}}=\frac{z \bar{\theta}}{(1-z \bar{z})^{2}}, \\
& \omega_{\theta \bar{\theta}}=\frac{1-z \bar{z}-2 \theta \bar{\theta}}{(1-z \bar{z}-\theta \bar{\theta})^{2}}=\frac{1}{(1-z \bar{z})} .
\end{aligned}
$$

It is clear from these explicit formulas that $\omega$ is non-degenerate and closed and thus supersymplectic.

Observe that

$$
\operatorname{Ber}\left(\begin{array}{ll}
\omega_{z \bar{z}} & \omega_{z \bar{\theta}} \\
\omega_{\theta \bar{z}} & \omega_{\theta \bar{\theta}}
\end{array}\right)=(1-Z \bar{Z})^{-1}
$$


As explained in the introduction, a super Poisson bracket may be constructed out of $\omega$. Explicitly, using (II.24) we obtain, for $f, g \in C^{\infty}(\mathscr{U})$,

$$
\begin{aligned}
\{f, g\}= & -(1-z \bar{z}-\theta \bar{\theta})(1-z \bar{z})\left(\partial_{z} f \partial_{\bar{z}} g-\partial_{\bar{z}} f \partial_{z} g\right) \\
& +(1-z \bar{z}) z \bar{\theta}\left((-1)^{p(f)} \partial_{z} f \partial_{\bar{\theta}} g-\partial_{\bar{\theta}} f \partial_{z} g\right) \\
& +(1-z \bar{z}) \bar{z} \theta\left(\partial_{\theta} f \partial_{\bar{z}} g-(-1)^{p(f)} \partial_{\bar{z}} f \partial_{\theta} g\right) \\
& +(1-z \bar{z}-z \bar{z} \theta \bar{\theta})(-1)^{p(f)+1}\left(\partial_{\theta} f \partial_{\bar{\theta}} g+\partial_{\bar{\theta}} f \partial_{\theta} g\right) .
\end{aligned}
$$

This super Poisson bracket is $S U(1,1 \mid 1)$-invariant, as a consequence of the invariance of $\omega$. We have thus proven the following theorem.

Theorem II.4. The pair $\left(C^{\infty}(\mathscr{U}),\{\cdot, \cdot\}\right)$ is a Poisson superalgebra with a $S U(1,1 \mid 1)$ invariant super Poisson bracket.

II.D. Define the following form ("super Poincaré measure"),

$$
d \mu(Z):=\frac{1}{\pi}(1-Z \bar{Z})^{-1} d^{2} z d^{2} \theta,
$$

where $d^{2} z=(i / 2) d z \wedge d \bar{z}$ and $d^{2} \theta=\mathrm{d} \theta \wedge d \bar{\theta}$. As a simple consequence of (II.17), we obtain the following proposition.

Proposition II.5. The form (II.26) is $S U(1,1 \mid 1)$-invariant.

\section{Quantization of $U^{1 / 1}$}

III.A. The main object of this paper is the following perturbation of (II.26). For $r \geqq 2$ we set

$$
d \mu_{r}(Z):=(1-Z \bar{Z})^{r} d \mu(Z)=\frac{1}{\pi}(1-Z \bar{Z})^{r-1} d^{2} z d^{2} \theta
$$

Proposition III.1. The form (III.1) has the properties:

$$
\begin{aligned}
\int_{\mathscr{U}} d \mu_{r}(Z) & =1, \\
d \mu_{r}(\gamma(Z)) & =\gamma^{\prime}(Z)^{r} \overline{\gamma^{\prime}(Z)^{r}} d \mu_{r}(Z) .
\end{aligned}
$$

Remark. Since $r$ does not need to be an integer, the $r^{\text {th }}$ power of $\gamma^{\prime}(Z)$ in the above formula has to be defined carefully. Using Proposition II.1, we can write

$$
\gamma^{\prime}(Z)=(a z+b+\varepsilon \theta)^{-1}=(a z+b)^{-1}-(a z+b)^{-2} \varepsilon \theta
$$

with $a, b$ even and $\varepsilon$ odd. We now define

$$
\log \gamma^{\prime}(Z):=-\log (a z+b)+(a z+b)^{-1} \varepsilon \theta,
$$

where $\log z$ is a fixed branch of the $\operatorname{logarithm}$ (for concreteness: $\log z:=\log |z|$ $+i \arg z$, where $-\pi<\arg z \leqq \pi)$, and set $\gamma^{\prime}(Z)^{r}:=\exp \left\{r \log \gamma^{\prime}(Z)\right\}$.

Proof. The second statement is a consequence of Proposition II.2 and Proposition II.5. The first statement is remarkable in that the integral is independent of $r$, even 
though the normalization constant in (III.1) does not involve $r$. This is a manifestation of supersymmetry and comes about as follows. We have the expansion

$$
(1-z \bar{z}-\theta \bar{\theta})^{r-1}=(1-z \bar{z})^{r-1}-(r-1)(1-z \bar{z})^{r-2} \theta \bar{\theta},
$$

and so

$$
\int_{\mathscr{U}} d \mu_{r}(Z)=\frac{r-1}{\pi} \int_{U}(1-z \bar{z})^{r-2} d^{2} z=(r-1) \int_{0}^{1}(1-t)^{r-2} d t=1 .
$$

III.B. For $f, g \in C_{b}^{\infty}(\mathscr{U})$, we set

$$
(f, g)_{r}:=\int_{\mathscr{U}} \overline{f(Z)} g(Z) d \mu_{r}(Z) .
$$

For $f$ and $g$ arbitrary, (III.7) is not positive definite. When restricted to superholomorphic functions, $(f, g)_{r}$ turns out to be positive definite and so it defines an inner product. The completion of the resulting inner product space in the norm $\|\cdot\|_{r}$ induced by $(\cdot, \cdot)_{r}$ is a Hilbert space and we denote it by $\mathscr{H}_{r}(\mathscr{U})$.

Because the measure used to define the norm involves Grassmann integration, we must be careful about applying the usual analytic facts. The following propositions show that we can extract sup norms from an integral over $d \mu_{r}(Z)$, just as we could from an ordinary integral. In the following, $\|f\|_{0}:=\sup _{z \in U}|f(z)|$ denotes the usual sup norm of a bounded function $f$ on $U$.

Proposition III.2. For $\psi, \phi \in \mathscr{H}_{r}(\mathscr{U})$, and $f \in C_{b}^{\infty}(\mathscr{U})$ such that $f(Z)=f_{00}(z)$, we have

$$
\left|\int_{\mathscr{U}} \overline{\psi(Z)} f(Z) \phi(Z) d \mu_{r}(Z)\right| \leqq\left\|f_{00}\right\|_{0}\|\psi\|_{r}\|\phi\|_{r} .
$$

Proof. To simplify the notation, we will suppress the subscript $r$ in $\|\cdot\|_{r}$. First, note that in terms of components,

$$
\|\phi\|^{2}=\frac{r-1}{\pi} \int_{U}\left|\phi_{0}(z)\right|^{2}(1-z \bar{z})^{r-2} d^{2} z+\frac{1}{\pi} \int_{U}\left|\phi_{1}(z)\right|^{2}(1-z \bar{z})^{r-1} d^{2} z .
$$

Since $f(Z)=f_{00}(z)$, we have

$$
\begin{aligned}
\int_{\mathscr{U}} \overline{\psi(Z)} f_{00}(z) \phi(Z) d \mu_{r}(Z)= & \frac{1}{\pi} \int_{U} \overline{\psi_{1}(z)} f_{00}(z) \phi_{1}(z)(1-z \bar{z})^{r-1} d^{2} z \\
& +\frac{r-1}{\pi} \int_{U} \overline{\psi_{0}(z)} f_{00}(z) \phi_{0}(z)(1-z \bar{z})^{r-2} d^{2} z
\end{aligned}
$$

and so

$$
\begin{aligned}
& \left|\int_{\mathscr{U}} \overline{\psi(Z)} f_{00}(z) \phi(Z) d \mu_{r}(Z)\right| \\
& \leqq \\
& \qquad f_{00} \|_{0}\left[\frac{1}{\pi}\left\{\int_{U}\left|\psi_{1}(z)\right|^{2}(1-z \bar{z})^{r-1} d^{2} z\right\}^{1 / 2}\left\{\int_{U}\left|\phi_{1}(z)\right|^{2}(1-z \bar{z})^{r-1} d^{2} z\right\}^{1 / 2}\right. \\
& \left.\quad+\frac{r-1}{\pi}\left\{\int_{U}\left|\psi_{0}(z)\right|^{2}(1-z \bar{z})^{r-2} d^{2} z\right\}^{1 / 2}\left\{\int_{U}\left|\phi_{0}(z)\right|^{2}(1-z \bar{z})^{r-2} d^{2} z\right\}^{1 / 2}\right] .
\end{aligned}
$$


In view of (III.9), this implies that

$$
\left|\int_{\mathscr{U}} \overline{\psi(Z)} f(Z) \phi(Z) d \mu_{r}(Z)\right| \leqq\left\|f_{00}\right\|_{0}\|\psi\|\|\phi\|,
$$

and the claim is proven.

Proposition III.3. For $\psi, \phi \in \mathscr{H}_{\boldsymbol{r}}(\mathscr{U})$, and $f \in C_{b}^{\infty}(\mathscr{U})$, we have

$$
\left|\int_{\mathscr{U}} \overline{\psi(Z)} f(Z) \phi(Z) d \mu_{r}(Z)\right| \leqq \sum_{0 \leqq j, k \leqq 1} r^{-(j+k) / 2}\left\|f_{j k}\right\|_{0}\|\psi\|_{r}\|\phi\|_{r} .
$$

Proof. The claim is an immediate consequence of Proposition III. 2 and the following inequality,

$$
\|\theta \phi\| \leqq r^{-1 / 2}\|\phi\| .
$$

To prove (III.14), we write $\phi_{0}(z)=\sum_{n \geqq 0} a_{n} z^{n}$ and compute:

$$
\begin{aligned}
\|\theta \phi\|^{2} & =\frac{1}{\pi} \int_{U}\left|\phi_{0}(z)\right|^{2}\left(1-|z|^{2}\right)^{r-1} d^{2} z=\sum_{n \geqq 0}\left|a_{n}\right|^{2} \int_{0}^{1} \rho^{n}(1-\rho)^{r-1} d \rho \\
& =\sum_{n \geqq 0}\left|a_{n}\right|^{2} \frac{n ! \Gamma(r)}{\Gamma(n+r+1)} \leqq r^{-1} \sum_{n \geqq 0}\left|a_{n}\right|^{2} \frac{n ! \Gamma(r)}{\Gamma(n+r)} \\
& =r^{-1} \frac{r-1}{\pi} \int_{U}\left|\phi_{0}(z)\right|^{2}\left(1-|z|^{2}\right)^{r-2} d^{2} z \leqq r^{-1}\|\phi\|^{2},
\end{aligned}
$$

where we have used (III.9).

For future reference we formulate the following proposition, whose proof is an immediate consequence of Proposition III.3 and (II.2).

Proposition III.4. For $\psi, \phi \in \mathscr{H}_{r}(\mathscr{U})$, and $f \in C_{b}^{\infty}(\mathscr{U})$, we have

$$
\left|\int_{\mathscr{U}} \overline{\psi(Z)} f(Z) \phi(Z) d \mu_{r}(Z)\right| \leqq\|f\|_{0}\|\psi\|_{r}\|\phi\|_{r} .
$$

III.C. The Hilbert space $\mathscr{H}_{\boldsymbol{r}}(\mathscr{U})$ carries a natural projective unitary representation of the supergroup $S U(1,1 \mid 1)$. This is given by $\gamma \rightarrow U(\gamma)$, where

$$
U(\gamma) \phi(Z)=\left\{\left(\gamma^{-1}\right)^{\prime}(Z)\right\}^{r} \phi\left(\gamma^{-1}(Z)\right) .
$$

Proposition III.5. Formula (III.16) defines a projective unitary representation of $S U(1,1 \mid 1)$ on $\mathscr{H}_{\boldsymbol{r}}(\mathscr{U})$.

Proof. Set

$$
\sigma\left(\gamma_{1}, \gamma_{2}\right):=\frac{\left(\left(\left(\gamma_{1} \gamma_{2}\right)^{-1}\right)^{\prime}(Z)\right)^{r}}{\left(\gamma_{1}^{-1}\right)^{\prime}(Z)^{r}\left(\gamma_{2}^{-1}\right)^{\prime}\left(\gamma_{1}^{-1}(Z)\right)^{r}}
$$

We verify easily that $\sigma\left(\gamma_{1}, \gamma_{2}\right)$ is indeed independent of $Z$ (as our notation suggests). As a consequence,

$$
U\left(\gamma_{1} \gamma_{2}\right)=\sigma\left(\gamma_{1}, \gamma_{2}\right) U\left(\gamma_{1}\right) U\left(\gamma_{2}\right)
$$


From the independence of (III.17) on $Z$ we easily derive the following cocycle condition:

$$
\sigma\left(\gamma_{2}, \gamma_{3}\right) \sigma\left(\gamma_{1} \gamma_{2}, \gamma_{3}\right)^{-1} \sigma\left(\gamma_{1}, \gamma_{2} \gamma_{3}\right) \sigma\left(\gamma_{1}, \gamma_{2}\right)^{-1}=1
$$

which shows that (III.16) is consistent with associativity. The unitarity is a consequence of Proposition III.1.

III.D. We now define a projection map $P$ taking $C_{b}^{\infty}(\mathscr{U})$ to $\mathscr{H}_{r}(\mathscr{U})$.

Proposition III.6. For $f \in C_{b}^{\infty}(\mathscr{U})$, set

$$
P f(Z):=\int_{\mathscr{U}} K^{r}(Z, W) f(W) d \mu_{r}(W),
$$

where

$$
K^{r}(Z, W)=(1-Z \bar{W})^{-r}
$$

Then $P f \in \mathscr{H}_{r}(\mathscr{U})$ and $P f=f$, if $f \in \mathscr{H}_{r}(\mathscr{U})$.

Proof. We verify easily that the sequence $\left\{\phi_{n, j}\right\}_{n \geqq 0, j=0,1}$, where

$$
\begin{aligned}
& \phi_{n, 0}(Z)=\left(\frac{\Gamma(n+r)}{\Gamma(r) n !}\right)^{1 / 2} z^{n}, \\
& \phi_{n, 1}(Z)=\left(\frac{\Gamma(n+r+1)}{\Gamma(r) n !}\right)^{1 / 2} z^{n} \theta,
\end{aligned}
$$

forms an orthonormal basis for $\mathscr{H}_{r}(\mathscr{U})$. Consequently,

$$
K^{r}(Z, W):=\sum_{n, j} \phi_{n, j}(Z) \overline{\phi_{n, j}(W)}=(1-Z \bar{W})^{-r}
$$

is the Bergman (or reproducing) kernel for $\mathscr{H}_{\boldsymbol{r}}(\mathscr{U})$ and the claim follows.

To each point $Z \in \mathscr{U}$, we will associate an element $\gamma_{Z}$ of $S U(1,1 \mid 1)$, such that $\gamma_{Z}$ maps the origin in $\mathscr{U}$ to the point $Z$. This element is defined by

$$
\gamma_{Z}:=c^{-1}\left(\begin{array}{ccc}
1 & z & c^{-1}(\theta-z \bar{\theta}) \\
\bar{z} & 1 & c^{-1}(\bar{z} \theta-\bar{\theta}) \\
\bar{\theta} & \theta & c^{-1}(1-z \bar{z})
\end{array}\right)
$$

where

$$
c=(1-z \bar{z}-\theta \bar{\theta})^{1 / 2} .
$$

For future reference, the action of $\gamma_{Z}$ on $W=(w, \eta) \in \mathscr{U}$ is given explicitly by

$$
\begin{aligned}
& \gamma_{Z}(W)_{0}=z+\frac{1-z \bar{z}}{1+\bar{z} w} w+\frac{(1-z \bar{z})^{1 / 2}}{1+\bar{z} w} \theta \eta-\frac{(1-z \bar{z})^{1 / 2}}{(1+\bar{z} w)^{2}}(\bar{z} \theta-\bar{\theta}) w \eta, \\
& \gamma_{Z}(W)_{1}=\theta+\frac{\bar{\theta}-\bar{z} \theta}{1+\bar{z} w} w+\frac{(1-z \bar{z}-\theta \bar{\theta})^{1 / 2}}{1+\bar{z} w} \eta .
\end{aligned}
$$

The Bergman kernel given by (III.21) has the following properties with respect to $S U(1,1 \mid 1)$. 
Proposition III.7. The Bergman kernel transforms under the action of $S U(1,1 \mid 1)$ according to

$$
K^{r}(\gamma(Z), \gamma(W))=\gamma^{\prime}(Z)^{-r}{\overline{\gamma^{\prime}(W)}}^{-r} K(Z, W) .
$$

Furthermore, for $K^{r}(Z, Z)$ we have

$$
K^{r}(Z, Z)=\gamma_{Z}^{\prime}(0)^{-r} \overline{\gamma_{Z}^{\prime}(0)^{-r}} .
$$

Proof. The first statement follows immediately from Proposition II.2. The second is a direct consequence of the first and the fact that $K^{r}(0,0)=1$.

III.E. For $f \in C_{b}^{\infty}(\mathscr{U})$ and $\phi \in \mathscr{H}_{r}(\mathscr{U})$, we set

$$
\left(T_{r}(f) \phi\right)(Z):=\int_{\mathscr{U}} K^{r}(Z, W) f(W) \phi(W) d \mu_{r}(W) .
$$

Proposition III.8. $T_{r}(f)$ is a bounded operator on $\mathscr{H}_{r}(\mathscr{U})$. Furthermore,

$$
\left\|T_{r}(f)\right\| \leqq \sum_{0 \leqq j, k \leqq 1} r^{-(j+k) / 2}\left\|f_{j k}\right\|_{0} .
$$

Proof. Clearly, $\left(T_{r}(f) \phi\right)(Z)$ is holomorphic. From the reproducing property of $K^{r}(Z, W)$,

$$
\left(\phi, T_{r}(f) \psi\right)=\int_{\mathscr{U}} \overline{\phi(Z)} f(Z) \psi(Z) d \mu_{r}(Z)
$$

The inequality follows immediately from Proposition III.3.

We call the operator $T_{r}(f)$ a super Toeplitz operator with symbol $f$, and we let $\mathscr{T}_{r}^{\infty}(\mathscr{U})$ denote the *-algebra generated by all super Toeplitz operators with bounded symbols. Observe that

$$
T_{r}(f \circ \gamma)=U(\gamma)^{-1} T_{r}(f) U(\gamma),
$$

where $U(\gamma)$ is defined by (III.16).

III.F. We now come to the main result of this section, which is that the map $C_{b}^{\infty}(\mathscr{U}) \rightarrow \mathscr{T}_{r}^{\infty}(\mathscr{U})$ given by $T_{r}$ is a deformation quantization. Define

$$
\Gamma_{j k}(Z):=\left.\frac{\partial}{\partial W_{j}} \gamma_{Z}(W)_{k}\right|_{W=0} .
$$

In Sect. V, we will prove the following two theorems.

Theorem III.9. For $f \in C_{b}^{\infty}(\mathscr{U})$, we have

$$
\lim _{r \rightarrow \infty}\left\|T_{r}(f)\right\|_{r}=\left\|f_{00}\right\|_{0} .
$$

The above theorem is a rather peculiar fact, showing that the classical limit of $T_{r}(f)$ "forgets" about the fermionic degrees of freedom. 
Theorem III.10. For $f, g \in C_{b}^{\infty}(\mathscr{U})$, with the components $f_{\alpha \beta}$ compactly supported, there is a constant $C=C(f, g)$ (depending on $f$ and $g$ ), such that

$\left\|T_{r}(f) T_{r}(g)-T_{r}(f g)-r^{-1} \sum_{j, k, l}(-1)^{j p(f)+1} T_{r}\left(\bar{\Gamma}_{l j} \Gamma_{l k} \partial_{k} f \bar{\partial}_{j} g\right)\right\|_{r} \leqq C r^{-2}$,

for $r$ sufficiently large.

As a consequence of this theorem, we conclude that $\mathscr{T}_{r}^{\infty}(\mathscr{U})$ is a quantum deformation of the Poisson superalgebra $C_{b}^{\infty}(\mathscr{U})$, with the ratio $\mu=(r-1)^{-1}$ playing the role of Planck's constant. The assumption that $f$ has a compact support is certainly not optimal and we regard it as a proviso. However, a closer look at our proof shows that some kind of decay of at least one symbol at the boundary of $U$ is needed. On the other hand, it is easy to verify that for polynomial $f$ and $g$, the conclusion of the theorem remains valid.

Theorem III.11. Under the assumptions of Theorem III.10,

$$
\left\|r\left[T_{r}(f), T_{r}(g)\right]-T_{r}(\{f, g\})\right\|_{r} \leqq C r^{-1},
$$

for $r$ sufficiently large.

Proof. We see from (III.26) that

$$
\begin{aligned}
& \Gamma_{00}(Z)=1-z \bar{z}, \\
& \Gamma_{10}(Z)=-(1-z \bar{z})^{1 / 2} \theta, \\
& \Gamma_{01}(Z)=\bar{\theta}-\bar{z} \theta, \\
& \Gamma_{11}(Z)=(1-z \bar{z}-\theta \bar{\theta})^{1 / 2} .
\end{aligned}
$$

The proof follows immediately from Theorem III.10 and the explicit form (II.25) of the super Poisson bracket.

\section{The $\mathbb{C}^{*}$-Algebra of Super Toeplitz Operators}

$I V . A$. In this section we study the structure of the $\mathbb{Z}_{2}$-graded $\mathbb{C}^{*}$-algebra $\mathscr{T}_{r}(\overline{\mathscr{U}})$ generated by all super Toeplitz operators with symbols $f$ whose components $f_{j k}$ extend to $C^{\infty}$-functions on the closure of the unit disc $U$. This $\mathbb{C}^{*}$-algebra is generated by $\sigma:=T_{r}(z), \bar{\sigma}:=T_{r}(\bar{z})=\sigma^{*}, \chi:=T_{r}(\theta)$, and $\bar{\chi}:=T_{r}(\bar{\theta})=\chi^{*}$.

Proposition IV.1. The generators of $\mathscr{T}_{\boldsymbol{r}}(\overline{\mathscr{U}})$ satisfy the following relations,

$$
\begin{aligned}
& {[\bar{\sigma}, \sigma]=\mu(I-\sigma \bar{\sigma}-\chi \bar{\chi})(I-\bar{\sigma} \sigma),} \\
& {[\sigma, \bar{\chi}]=\mu(I-\bar{\sigma} \sigma) \sigma \bar{\chi},} \\
& {[\chi, \bar{\chi}]=\mu(I-\bar{\sigma} \sigma-\bar{\sigma} \sigma \chi \bar{\chi}),} \\
& {[\sigma, \chi]=0,} \\
& {[\chi, \chi]=0,}
\end{aligned}
$$

and their hermitian conjugates, where $\mu:=(r-1)^{-1}$. 
Proof. The easiest way to obtain these relations is to observe that

$$
\begin{aligned}
& \sigma \phi_{n, k}(Z)=\left(\frac{n+1}{n+r+k}\right)^{1 / 2} \phi_{n+1, k}(\mathrm{Z}), \\
& \bar{\sigma} \phi_{n, k}(Z)=\left(\frac{n}{n+r+k-1}\right)^{1 / 2} \phi_{n-1, k}(Z), \\
& \chi \phi_{n, k}(Z)=(1-k)(n+r)^{-1 / 2} \phi_{n, k+1}(Z), \\
& \bar{\chi} \phi_{n, k}(Z)=k(n+r)^{-1 / 2} \phi_{n, k-1}(Z),
\end{aligned}
$$

where $\left\{\phi_{n, k}\right\}$ is the orthonormal basis defined by (III.22).

$I V$.B. Let $\mathscr{K}$ denote the $\mathbb{Z}_{2}$-graded $\mathbb{C}^{*}$-algebra of compact operators on $\mathscr{H}_{r}(\mathscr{U})$, and let $C\left(S^{1}\right)$ be the trivially $\mathbb{Z}_{2}$-graded $\mathbb{C}^{*}$-algebra of continuous functions on the unit circle. The following theorem describes the structure of $\mathscr{T}_{r}(\overline{\mathscr{U}})$; namely it states that $\mathscr{T}_{r}(\overline{\mathscr{U}})$ is an extension of $C\left(S^{1}\right)$ by $\mathscr{K}$.

Theorem IV.2. There is a short exact sequence of $\mathbb{Z}_{2}$-graded $\mathbb{C}^{*}$-algebras,

$$
0 \rightarrow \mathscr{K} \rightarrow \mathscr{T}_{r}(\overline{\mathscr{U}}) \rightarrow C\left(S^{1}\right) \rightarrow 0 .
$$

Furthermore, as $\mathbb{C}^{*}$-algebras, $\mathscr{T}_{r}(\overline{\mathscr{U}}) \simeq \mathscr{T}_{s}(\overline{\mathscr{U}})$, for all $r, s>1$.

Proof. Let $\mathscr{I}$ denote the commutator ideal in $\mathscr{T}_{r}(\overline{\mathscr{U}})$. It follows immediately from (IV.1) that the quotient $\mathbb{C}^{*}$-algebra $\mathscr{T}_{r}(\overline{\mathscr{U}}) / \mathscr{I}$ is generated by a single element and so $\mathscr{T}_{r}(\overline{\mathscr{U}}) / \mathscr{I} \simeq C\left(S^{1}\right)$. We claim that $\mathscr{I} \simeq \mathscr{K}$. Indeed, as a consequence of (IV.1) and (IV.2), $[\bar{\sigma}, \sigma] \in \mathscr{K}$. Furthermore, from (IV.2), $\chi \in \mathscr{K}$ and so all the commutators involving $\chi$ and $\bar{\chi}$ are compact. This shows that $\mathscr{I} \subset \mathscr{K}$. On the other hand, since $\mathscr{T}_{r}(\overline{\mathscr{U}})$ is irreducible, Theorem 5.39 in [11] implies that $\mathscr{K} \subset \mathscr{T}_{r}(\overline{\mathscr{U}})$. Consequently, $\mathscr{I}$ is a non-zero ideal in $\mathscr{K}$ and so it must be equal to $\mathscr{K}$. This proves (IV.3).

To prove the second statement, we realize the $\mathbb{C}^{*}$-algebras $\mathscr{T}_{r}(\overline{\mathscr{U}})$ and $\mathscr{T}_{s}(\overline{\mathscr{U}})$ on the $\mathbb{Z}_{2}$-graded Hilbert space $l^{2}\left(\mathbb{Z}_{+}\right) \oplus l^{2}\left(\mathbb{Z}_{+}\right)$and denote the corresponding generators by $\sigma_{r}, \sigma_{s}$, etc. Obviously, $\chi_{r}, \sigma_{s}-\sigma_{r} \in \mathscr{K} \subset \mathscr{T}_{s}(\overline{\mathscr{U}})$ and so $\mathscr{T}_{r}(\overline{\mathscr{U}}) \subset \mathscr{T}_{s}(\overline{\mathscr{U}})$. By the same argument, $\mathscr{T}_{s}(\overline{\mathscr{U}}) \subset \mathscr{T}_{r}(\overline{\mathscr{U}})$, and the claim follows.

$I V . C$. We now show that the $\mathbb{C}^{*}$-algebra $\mathscr{T}_{r}(\overline{\mathscr{U}})$ can be characterized in terms of generators and relations. Let $\mathscr{P}_{r}$ be the unital algebra generated by $\sigma, \bar{\sigma}, \chi, \bar{\chi}$ with relations (IV.1). A representation $\pi: \mathscr{P}_{r} \rightarrow \mathscr{L}(\mathscr{H})$ of $\mathscr{P}_{r}$ on a Hilbert space $\mathscr{H}$ is called a $*$-representation, if $\pi(\sigma)^{*}=\pi(\bar{\sigma})$ and $\pi(\chi)^{*}=\pi(\bar{\chi})$.

Theorem IV.3. Let $\pi: \mathscr{P}_{r} \rightarrow \mathscr{L}(\mathscr{H})$ be an irreducible *-representation of $\mathscr{P}_{r}$ on a Hilbert space $\mathscr{H}$. Then, $\pi$ is unitarily equivalent to either the one dimensional representation $\mathscr{H}=\mathbb{C}$, with

$$
\pi(\chi)=0, \quad \pi(\sigma)=e^{i \theta},
$$

for some $\theta \in[0,2 \pi)$, or to the representation (IV.2).

Proof. Let $\pi$ be an irreducible $*$-representation of $\mathscr{P}_{r}$ on a Hilbert space $\mathscr{H}$. Consider first the subspace

$$
W=\operatorname{Ker}(1-\bar{\sigma} \sigma) \cap \operatorname{Ker}(\bar{\chi}) .
$$


Using the commutation relations, it is easy to see that $W$ is an invariant, closed subspace for the representation $\pi$, and so either $W=\mathscr{H}$ or $W=0$. If $W=\mathscr{H}$, then the representation $\pi$ is one dimensional and it is unitarily equivalent to a representation of the type (IV.4).

Assume from now on that $W=0$ and set $\mathscr{H}_{0}:=\operatorname{Ker}(\bar{\chi})$. It is easy to see that $\mathscr{H}_{0}$ cannot be zero. Let $\mathscr{S}_{r}$ be the subalgebra of $\mathscr{P}_{r}$ generated by $\sigma$ and $\bar{\sigma}$ with the commutation relation

$$
[\bar{\sigma}, \sigma]=\frac{1}{r-1}(1-\sigma \bar{\sigma})(1-\bar{\sigma} \sigma) .
$$

The representation $\pi$ induces a representation $\pi_{0}$ of $\mathscr{S}_{r}$ on $\mathscr{H}_{0}$. We claim that this representation is irreducible. Indeed, suppose we have an invariant proper subspace $V_{0} \subset \mathscr{H}_{0}$. Pick a non-zero vector $\xi \in \mathscr{H}_{0}, \xi \notin V_{0}$. The spaces $V_{0}$ and $\chi\left(V_{0}\right)$ are clearly orthogonal. Consider now the orthogonal sum $V_{0} \oplus \chi\left(V_{0}\right)$. Using the algebra structure, it is easy to show that the representation $\pi$ restricts to a representation of $\mathscr{P}_{r}$ on $V_{0} \oplus \chi\left(V_{0}\right)$. It is clear that $\xi \notin \chi\left(V_{0}\right)$, for $\chi\left(V_{0}\right)$ is orthogonal to $\operatorname{Ker}(\bar{\chi})$. This shows that $\pi_{0}$ is irreducible. At the same time, the above argument shows that

$$
\mathscr{H}=\mathscr{H}_{0} \oplus \mathscr{H}_{1},
$$

where $\mathscr{H}_{1}=\chi\left(\mathscr{H}_{0}\right)$. Now we can invoke Theorem III.2 of [12]: there is an orthonormal basis $\phi_{n, 0}, n=0,1, \ldots$, for $\mathscr{H}_{0}$ such that $\sigma$ and $\bar{\sigma}$ act according to (IV.2).

Now, using this result it is easy to check that

$$
r(1-\bar{\sigma} \sigma)=(r-1)(1-\sigma \bar{\sigma}(1-\mu(1-\bar{\sigma} \sigma))),
$$

on $\mathscr{H}_{0}$. The relation, $\chi \bar{\sigma}-\bar{\sigma} \chi=\mu \chi \bar{\sigma}(1-\bar{\sigma} \sigma)$, yields

$$
r \chi(1-\bar{\sigma} \sigma)=(r-1)(1-\sigma \bar{\sigma}) \chi,
$$

on $\mathscr{H}_{0}$. Finally, we have

$$
[\bar{\sigma}, \sigma] \chi=\mu(1-\bar{\sigma} \sigma)[(1-\sigma \bar{\sigma}) \chi-\mu \chi(1-\bar{\sigma} \sigma)],
$$

on $\mathscr{H}_{0}$, which by means of (IV.7) can be rewritten as

$$
[\bar{\sigma}, \sigma] \chi=\frac{1}{r}(1-\bar{\sigma} \sigma)(1-\sigma \bar{\sigma}) \chi .
$$

This implies the following relation on $\mathscr{H}_{1}$ :

$$
[\bar{\sigma}, \sigma]=\frac{1}{r}(1-\bar{\sigma} \sigma)(1-\sigma \bar{\sigma}) .
$$

As a consequence, $\pi$ induces a representation $\pi_{1}$ of $\mathscr{S}_{r+1}$ on $\mathscr{H}_{1}$.

We now show that $\pi_{1}$ is irreducible. To prove this, observe first that $\left.\chi\right|_{\mathscr{H}_{0}}$ is injective. Indeed, if a vector $\phi$ is in the kernels of both $\chi$ and $\bar{\chi}$, then the commutation relation $[\chi, \bar{\chi}]=\mu(I-\bar{\sigma} \sigma-\bar{\sigma} \sigma \bar{\chi} \chi)$ implies that $(1-\bar{\sigma} \sigma) \phi=0$. But this is impossible because $W=0$. Let now $V_{1} \subset \mathscr{H}_{1}$ be a closed, invariant subspace. It is then easy to verify that $\chi^{-1}\left(V_{1}\right) \in \mathscr{H}_{0}$ is an invariant subspace for the 
representation $\pi_{0}$ of $\mathscr{S}_{r}$, and so $\chi^{-1}\left(V_{1}\right)=\mathscr{H}_{0}$. By the injectivity of $\chi$, we conclude $V_{1}=\mathscr{H}_{1}$. We use Theorem III.2 of [12] to determine the action of $\mathscr{S}_{r+1}$ on $\mathscr{H}_{1}$. There is an orthonormal basis $\phi_{n, 1}$ for $\mathscr{H}_{1}$ and the action of $\mathscr{S}_{r+1}$ on the elements of this basis is given by (IV.2). Finally, the commutation relation of $\bar{\sigma}$ with $\chi$ tells that $\chi\left(\operatorname{Ker} \bar{\sigma} \cap \mathscr{H}_{0}\right)=\operatorname{Ker}\left(\bar{\sigma} \cap \mathscr{H}_{1}\right)$. Denoting by $\mathscr{H}_{n, j}$ the one dimensional space spanned by $\phi_{n, j}$, we easily see that $\chi: \mathscr{H}_{n, 0} \rightarrow \mathscr{H}_{n, 1}$. Furthermore, an explicit computation shows that $\left.\bar{\chi} \chi\right|_{\mathscr{H}_{n, 0}}=(n+r)^{-1} I$. This completes the proof of the theorem.

A standard consequence of the above two theorems is the following characterization of $\mathscr{T}_{r}(\overline{\mathscr{U}})$. Let $\mathscr{C}_{r}(\overline{\mathscr{U}})$ denote the universal enveloping $\mathbb{C}^{*}$-algebra of $\mathscr{P}_{r}$, i.e. the closure of $\mathscr{P}_{r} / \mathscr{N}$ in the norm $\|x\|:=\sup _{\pi}\{\|\pi(x)\|\}$, where the supremum is taken over all $*$-representations of $\mathscr{P}_{r}$, and where $\mathscr{N}:=\left\{x \in P_{r}:\|x\|=0\right\}$ is the nil-ideal.

Theorem IV.4. As $\mathbb{C}^{*}$-algebras, $\mathscr{T}_{r}(\overline{\mathscr{U}}) \simeq \mathscr{C}_{r}(\overline{\mathscr{U}})$.

Proof. The proof follows the proof of Theorem III.5 in [12].

\section{Proof of Deformation Estimates for $U^{1 / 1}$}

V.A. Proof of Theorem III.9. From Proposition III.8,

$$
\left\|T_{r}(f)\right\| \leqq\left\|f_{00}\right\|_{0}+O\left(r^{-1 / 2}\right),
$$

as $r \rightarrow \infty$, i.e. $\lim \sup _{r \rightarrow \infty}\left\|T_{r}(f)\right\| \leqq\left\|f_{00}\right\|_{0}$. We will show below that

$$
\left\|f_{00}\right\|_{0} \leqq\left\|T_{r}(f)\right\|+o(1) \text {, }
$$

as $r \rightarrow \infty$, i.e. $\liminf _{r \rightarrow \infty}\left\|T_{r}(f)\right\| \geqq\left\|f_{00}\right\|_{0}$, and this will prove the claim.

To prove (V.2), we set $Z=(z, 0)$ and write

$$
\begin{aligned}
f(Z)=f_{00}(z)= & \left(\phi_{0,0}, T\left(f \circ \gamma_{Z}\right) \phi_{0,0}\right) \\
& +\left\{f_{00}(z)-\int_{\mathscr{U}} f\left(\gamma_{Z}(W)\right) d \mu_{r}(W)\right\},
\end{aligned}
$$

where $\phi_{0,0}=1$. Using (III.32), (III.5), and (III.26), we rewrite the above equation as

$$
\begin{aligned}
f_{00}(z)= & \left(\phi_{0,0}, U\left(\gamma_{Z}\right)^{-1} T_{r}(f) U\left(\gamma_{Z}\right) \phi_{0,0}\right) \\
& +\left\{f_{00}(z)-\frac{r-1}{\pi} \int_{U} f_{00}\left(\frac{w+z}{1+\bar{z} w}\right)\left(1-|w|^{2}\right)^{r-2} d^{2} w\right\} \\
& +\frac{1}{\pi} \int_{U} f_{11}\left(\frac{w+z}{1+\bar{z} w}\right) \frac{1-|z|^{2}}{(1+\bar{z} w)^{2}}\left(1-|w|^{2}\right)^{r-1} d^{2} w .
\end{aligned}
$$

The first term in (V.4) can be bounded by $\left\|T_{r}(f)\right\|$, as $U\left(\gamma_{Z}\right)$ is unitary. By Lemma VI.3 of [12], the second term is $o(1)$ uniformly in $z$, as $r \rightarrow \infty$. Finally, using the inequality $\left|(1+\bar{z} w)^{-1}\right| \leqq(1-|w|)^{-1}$ we bound the third term by

$$
\frac{4}{\pi}\left\|f_{11}\right\|_{0} \int_{U}\left(1-|w|^{2}\right)^{r-3} d^{2} w=\frac{4}{r-2}\left\|f_{11}\right\|_{0},
$$

and the claim follows. 
V.B. Proof of Theorem III.10. Our procedure will be to expand [12]

$$
\left(\phi, T_{r}(f) T_{r}(g) \psi\right)=\int_{\mathscr{U} \times \mathscr{U}} \overline{\phi(Z)} f(Z) K^{r}(Z, X) g(X) \psi(X) d \mu_{r}(Z) d \mu_{r}(X),
$$

where $\psi, \phi \in \mathscr{H}_{r}(\mathscr{U})$ and $f, g \in C^{\infty}(\mathscr{U})$, in a power series in $r$. We make the substitution $X=\gamma_{Z}(W)$, and use the transformation properties of the Bergman kernel to rewrite (V.5) as

$\left(\phi, T_{r}(f) T_{r}(g) \psi\right)=\int_{\mathscr{U} \times \mathscr{U}} \overline{\phi(Z)} f(Z) \frac{K^{r}(Z, Z)}{K^{r}\left(\gamma_{Z}(W), Z\right)} g\left(\gamma_{Z}(W)\right) \psi\left(\gamma_{Z}(W)\right) d \mu_{r}(Z) d \mu_{r}(W)$.

The next step will be to expand $g\left(\gamma_{Z}(W)\right)$ in a Taylor series. We will expand out to fourth order, as follows:

$$
\begin{aligned}
g\left(\gamma_{Z}(W)\right)= & g(Z)+\sum_{j, l}\left(W_{l} \Gamma_{l j}(Z) \partial_{j} g(Z)+\overline{W_{l} \Gamma_{l j}(Z)} \bar{\partial}_{j} g(Z)\right) \\
& +\frac{1}{2} \sum_{j, k, l, m} W_{l} \Gamma_{l j}(Z) W_{k} \Gamma_{k m}(Z) \partial_{m} \partial_{j} g(Z)+\frac{1}{2} \sum_{j, l, m} W_{l} W_{m} \Gamma_{m l j}(Z) \partial_{j} g(Z) \\
& +\sum_{j, k, l, m} \overline{W_{l} \Gamma_{l j}(Z)} W_{m} \Gamma_{m k}(Z) \partial_{k} \bar{\partial}_{j} g(Z) \\
& +\frac{1}{2} \sum_{j, k, l, m} \overline{W_{l} \Gamma_{l j}(Z)} \overline{W_{m} \Gamma_{m k}(Z)} \bar{\partial}_{k} \bar{\partial}_{j} g(Z)+\frac{1}{2} \sum_{j, l, m} \overline{W_{l} W_{m} \Gamma_{m l j}(Z)} \bar{\partial}_{j} g(Z) \\
& +3 \operatorname{rd~order~terms~} \\
& +G(Z, W),
\end{aligned}
$$

where

$$
\begin{aligned}
\Gamma_{l j}(Z) & :=\left.\frac{\partial}{\partial W_{l}} \gamma_{Z}(W)_{j}\right|_{W=0}, \\
\Gamma_{m l j}(Z) & :=\left.\frac{\partial}{\partial W_{m}} \frac{\partial}{\partial W_{l}} \gamma_{Z}(W)_{j}\right|_{W=0},
\end{aligned}
$$

and where the fourth order remainder term is given by

$$
G(Z, W):=\frac{1}{3 !} \int_{0}^{1} d s(1-s)^{3} \frac{d^{4}}{d s^{4}} g\left(\gamma_{Z}(s W)\right) .
$$

Denote by $I_{p, q}$ the contribution to the integral from the term in the expansion of $g$ with $p$ powers of $W$ and $q$ powers of $\bar{W}$, and let $R$ denote the contribution of the remainder term. In evaluating these terms, we will make use of the following facts. Suppose we have a holomorphic function $h$ on $\mathscr{U}$. Then one can easily check that

$$
\int_{\mathscr{U}} \bar{w}^{k} h(W) d \mu_{r}(W)=\frac{1}{k !} \frac{\partial^{k} h}{\partial w^{k}}(0) \int_{\mathscr{U}}|w|^{2 k} d \mu_{r}(W),
$$

and

$$
\int_{\mathscr{U}} \bar{w}^{k} \bar{\eta} h(W) d \mu_{r}(W)=\frac{1}{k !} \frac{\partial^{k+1} h}{\partial \eta \partial w^{k}}(0) \int_{\mathscr{U}}|w|^{2 k} \bar{\eta} \eta d \mu_{r}(W),
$$

where $W=(w, \eta)$. 
For the lowest order term in the expansion, we have

$$
I_{0,0}=\int_{\mathscr{U} \times \mathscr{U}} \overline{\phi(Z)} f(Z) \frac{K^{r}(Z, Z)}{K^{r}\left(\gamma_{Z}(W), Z\right)} g(Z) \psi\left(\gamma_{Z}(W)\right) d \mu_{r}(Z) d \mu_{r}(W) .
$$

The integrand is holomorphic in $W$, so we apply (V.10) to get

$$
\begin{aligned}
I_{0,0} & =\int_{\mathscr{U}} \overline{\phi(Z)} f(Z) g(Z) \psi(Z) d \mu_{r}(Z) \\
& =\left(\phi, T_{r}(f g) \psi\right) .
\end{aligned}
$$

The same fact also easily implies that $I_{p, q}=0$ for $p>q$.

The next non-zero term is thus $I_{0,1}$, which is given by

$$
I_{0,1}=\sum_{j, l} \int_{\mathscr{U} \times \mathscr{U}} \overline{\phi(Z)} f(Z) \overline{W_{l} \Gamma_{l j}(Z)} \bar{\partial}_{j} g(Z) \frac{\psi\left(\gamma_{Z}(W)\right)}{K^{r}\left(\gamma_{Z}(W), Z\right)} d \mu(Z) d \mu_{r}(W),
$$

where we have incorporated the $K^{r}(Z, Z)$ term into the super Poincare measure $d \mu(Z)$, defined by (II.26). We now apply (V.10) and (V.11), using the integrals

$$
\int_{\mathscr{U}}|w|^{2} d \mu_{r}(W)=\int_{\mathscr{U}} \bar{\eta} \eta d \mu_{r}(W)=\frac{1}{r} .
$$

This gives

$$
I_{0,1}=\frac{1}{r} \sum_{j, l}(-1)^{\varepsilon_{l}\left(p(g)+\varepsilon_{j}\right)} \int_{\mathscr{U}} \overline{\phi(Z)} f(Z) \overline{\Gamma_{l j}(Z)} \bar{\partial}_{j} g(Z)\left[\frac{\partial}{\partial W_{l}} \frac{\psi\left(\gamma_{Z}(W)\right)}{K^{r}\left(\gamma_{Z}(W), Z\right)}\right]_{W=0} d \mu(Z),
$$

where $\varepsilon_{l}=p\left(Z_{l}\right)$, and the sign arises from the permutation of elements of the integrand (keeping in mind the fact that $\overline{W T}=\bar{\Gamma} \bar{W}$ ). Applying the chain rule gives

$$
\begin{aligned}
I_{0,1}= & \frac{1}{r} \sum_{j, k, l}(-1)^{\varepsilon_{l}\left(p(g)+\varepsilon_{j}\right)} \int_{\mathscr{U}} \overline{\phi(Z)} f(Z) \overline{\Gamma_{l j}(Z)} \bar{\partial}_{j} g(Z) \Gamma_{l k}(Z) \partial_{k}\left[\frac{\psi(Z)}{K^{r}(Z, Z)}\right] d \mu(Z) \\
= & \frac{1}{r} \sum_{j, k, l}(-1)^{\left(\varepsilon_{j}+\varepsilon_{k}\right) p(f)+\varepsilon_{k}\left(p(g)+\varepsilon_{j}\right)} \\
& \times \int_{\mathscr{U}} \overline{\phi(Z)} \overline{\Gamma_{l j}(Z)} \Gamma_{l k}(Z) f(Z) \bar{\partial}_{j} g(Z) \partial_{k}\left[\frac{\psi(Z)}{K^{r}(Z, Z)}\right] d \mu(Z)
\end{aligned}
$$

Next we integrate by parts,

$$
\begin{aligned}
I_{0,1}=-\frac{1}{r} \sum_{j, k, l}(-1)^{\varepsilon_{j} p(f)+\varepsilon_{k}\left(\varepsilon_{j}+1\right)} \int_{\mathscr{U}} \overline{\phi(Z)} \partial_{k}\left[\frac{\overline{\Gamma_{l j}(Z)} \Gamma_{l k}(Z)}{1-Z \bar{Z}} f(Z) \bar{\partial}_{j} g(Z)\right] \\
\times \psi(Z)(1-Z \bar{Z}) d \mu_{r}(Z)
\end{aligned}
$$




$$
\begin{aligned}
= & -\frac{1}{r} \sum_{j, k, l}(-1)^{\varepsilon_{j} p(f)+\varepsilon_{k}\left(\varepsilon_{j}+1\right)} \int_{\mathscr{U}} \overline{\phi(Z)} \partial_{k}\left[\frac{\overline{\Gamma_{l j}(Z)} \Gamma_{l k}(Z)}{1-Z \bar{Z}}\right] f(Z) \bar{\partial}_{j} g(Z) \\
& \times \psi(Z)(1-Z \bar{Z}) d \mu_{r}(Z) \\
& -\frac{1}{r} \sum_{j, k, l}(-1)^{\varepsilon_{j} p(f)} \int_{\mathscr{U}} \overline{\phi(Z)} \overline{\Gamma_{l j}(Z)} \Gamma_{l k}(Z) \partial_{k} f(Z) \bar{\partial}_{j} g(Z) \psi(Z) d \mu_{r}(Z) \\
& -\frac{1}{r} \sum_{j, k, l}(-1)^{\left(\varepsilon_{j}+\varepsilon_{k}\right) p(f)} \int_{\mathscr{U}} \overline{\phi(Z)} \overline{\Gamma_{l j}(Z)} \Gamma_{l k}(Z) f(Z) \partial_{k} \bar{\partial}_{j} g(Z) \psi(Z) d \mu_{r}(Z) .
\end{aligned}
$$

Observe that, as a consequence of the assumption that $r$ is sufficiently large, no boundary terms are present. One can quickly check that

$$
\sum_{k, l}(-1)^{\varepsilon_{k}\left(\varepsilon_{j}+1\right)} \partial_{k}\left[\frac{\overline{\Gamma_{l j}(Z)} \Gamma_{l k}(Z)}{1-Z \bar{Z}}\right]=0 .
$$

This leaves two terms in (V.18).

Now consider the term $I_{1,1}$, which is given by

$$
\begin{aligned}
I_{1,1}=\sum_{j, k, l, m} \int_{\mathscr{U} \times \mathscr{U}} & \overline{\phi(Z)} f(Z) \frac{K^{r}(Z, Z)}{K^{r}\left(\gamma_{Z}(W), Z\right)} \overline{W_{l} \Gamma_{l j}(Z)} W_{m} \Gamma_{m k}(Z) \partial_{k} \bar{\partial}_{j} g(Z) \\
& \times \psi\left(\gamma_{Z}(W)\right) d \mu_{r}(Z) d \mu_{r}(W) .
\end{aligned}
$$

Using (V.10) and (V.11), together with (V.15), we can perform the $W$ integration to get

$$
\begin{aligned}
I_{1,1} & =\frac{1}{r} \sum_{j, k, l} \int_{\mathscr{U}} \overline{\phi(Z)} f(Z) \overline{\Gamma_{l j}(Z)} \Gamma_{l k}(Z) \partial_{k} \overline{\partial_{j}} g(Z) \psi(Z) d \mu_{r}(Z) \\
& =\frac{1}{r} \sum_{j, k, l}(-1)^{\left(\varepsilon_{j}+\varepsilon_{k}\right) p(f)} \int_{\mathscr{U}} \overline{\phi(Z)} \overline{\Gamma_{l j}(Z)} \Gamma_{l k}(Z) f(Z) \partial_{k} \bar{\partial}_{j} g(Z) \psi(Z) d \mu_{r}(Z) .
\end{aligned}
$$

This exactly cancels the third term in (V.18), so that we finally obtain

$$
I_{0,1}+I_{1,1}=\frac{1}{r} \sum_{j, k, l}(-1)^{\varepsilon_{j} p(f)+1}\left(\phi, T_{r}\left(\bar{\Gamma}_{l j} \Gamma_{l k} \partial_{k} f \bar{\partial}_{j} g\right) \psi\right)
$$

Of the remaining second order terms, $I_{2,0}=0$, and $I_{0,2}$ is given by

$$
\begin{aligned}
I_{0,2}= & \frac{1}{2} \int_{\mathscr{U} \times \mathscr{U}} \overline{\phi(Z)} f(Z) K^{r}\left(\gamma_{Z}(W), Z\right)^{-1}\left[\sum_{j, k, l, m} \overline{W_{l} \Gamma_{l j}(Z)} \overline{W_{m} \Gamma_{m k}(Z)} \bar{\partial}_{k} \bar{\partial}_{j} g(Z)\right. \\
& \left.+\sum_{j, l, m} \overline{W_{l} W_{m} \Gamma_{m l j}(Z)} \bar{\partial}_{j} g(Z)\right] \psi\left(\gamma_{Z}(W)\right) d \mu(Z) d \mu_{r}(W)
\end{aligned}
$$

We want to bound this term for large r. First, we use (V.10) and (V.11) as before, to evaluate the $W$ integration. Noting that

$$
\int_{\mathscr{U}}|w|^{4} d \mu_{r}(W)=\frac{2}{r(r+1)},
$$


and

$$
\int_{\mathscr{U}}|w|^{2} \bar{\eta} \eta d \mu_{r}(W)=\frac{1}{r(r+1)},
$$

we can make the bound

$$
\begin{aligned}
\left|I_{0,2}\right| \leqq C r^{-2} \sum_{j, k, l, m} \mid \int_{\mathscr{U}} \overline{\phi(Z)} f(Z) & {\left[\overline{\Gamma_{l j}(Z) \Gamma_{m k}(Z)} \bar{\partial}_{k} \bar{\partial}_{j} g(Z)+\delta_{j k} \overline{\Gamma_{m l j}(Z)} \bar{\partial}_{k} g(Z)\right] } \\
\times & {\left[\frac{\partial^{2}}{\partial W_{l} \partial W_{m}} \frac{\psi\left(\gamma_{Z}(W)\right)}{K^{r}\left(\gamma_{Z}(W), Z\right)}\right]_{W=0} d \mu(Z) \mid . \quad(\text { V.2 }}
\end{aligned}
$$

As before, we can apply the chain rule to convert $W$ derivatives into $Z$ derivatives, and then integrate by parts to move these derivatives onto $f$ and $g$. Because of the compact support of $f$, the derivatives which hit the $\Gamma$ 's do not affect the result. We get a maximum of two derivatives of $f$ and four derivatives of $g$. Using Proposition III.4, we can extract the sup norms of the derivatives of $f$ and $g$ from the integral. In this way we obtain

$$
\left|I_{0,2}\right| \leqq C r^{-2}\|f\|_{t}\|g\|_{t}\|\psi\|\|\phi\|
$$

for some $t$.

We can apply the same arguments used for $I_{0,2}$ to the bound the third order terms. The terms are more complicated to write out than in the case of $I_{0,2}$, but the approach is exactly the same. The result is that

$$
\left|I_{p, q}\right| \leqq C r^{-2}\|f\|_{t}\|g\|_{t}\|\psi\|\|\phi\|,
$$

for some $t$ for $p+q=3$.

Now we turn to the remainder term, which is

$$
R=\int_{\mathscr{U} \times \mathscr{U}} \overline{\phi(Z)} f(Z) G(Z, W) \frac{\psi\left(\gamma_{Z}(W)\right)}{K^{r}\left(\gamma_{Z}(W), Z\right)} d \mu(Z) d \mu_{r}(W) .
$$

Note that

$$
\begin{aligned}
\frac{\psi\left(\gamma_{Z}(W)\right)}{K^{r}\left(\gamma_{Z}(W), Z\right)} & =\gamma_{Z}^{\prime}(W)^{r}{\overline{\gamma_{Z}^{\prime}(0)^{r}}}^{r}\left(\gamma_{Z}(W)\right), \\
& =K^{r}(Z, Z)^{-1 / 2} U\left(\gamma_{Z}^{-1}\right) \psi(W),
\end{aligned}
$$

where $U$ is the projective unitary representation of $S U(1,1 \mid 1)$ on $\mathscr{H}_{\boldsymbol{r}}(\mathscr{U})$, and we have used the fact that $\gamma_{Z}^{\prime}(0)$ is real. Denote $U\left(\gamma_{Z}^{-1}\right) \psi(W)$ by $\psi_{Z}(W)$, noting that $\left\|\psi_{Z}\right\|=\|\psi\|$. The remainder term can thus be written

$$
R=\int_{\mathscr{U} \times \mathscr{U}} \overline{\phi(Z)} K^{r}(Z, Z)^{1 / 2} f(Z) G(Z, W) \psi_{Z}(W) d \mu_{r}(Z) d \mu_{r}(W)
$$

We can write the components of the function $G(Z, W)$ as

$$
G(Z, W):=\sum_{0 \leqq j, k, l, m \leqq 1} G_{j k l m}(z, w) \bar{\theta}^{j} \theta^{k} \bar{\eta}^{l} \eta^{m} .
$$


For some positive integer $N$, we claim that we have the bound

$$
\sup _{z}\left|G_{j k l m}(z, w)\right| \leqq C\|g\|_{t} \frac{|w|^{4-l-m}}{\left(1-|w|^{2}\right)^{N}}
$$

for some $t$. This may be established as follows. Consider the definition of $G(Z, W)$, Eq. (V.9), which involves taking five derivatives, and the explicit form (III.26) of the function $\gamma_{Z}(W)$. First of all, each derivative with respect to $s$ in (V.9) brings out a factor of $W$, since only the combination $s W$ appears in the definition. This accounts for the $|w|^{4-l-m}$ appearing in (V.33).

One can see by inspection of the formulas (III.26) for $\gamma_{Z}(W)$ that divergence will come only from a denominator term of the form $1+s \bar{z} w$, raised to some power. Observe that such a term can be bounded using

$$
\begin{aligned}
|1+s \bar{z} w|^{-1} & \leqq(1-|w|)^{-1} \\
& \leqq 2\left(1-|w|^{2}\right)^{-1}
\end{aligned}
$$

which follows from $s \leqq 1,|z|<1,|w|<1$. One can check fairly quickly that the best bound one can make is $N=12$, but this will not really matter.

Lemma V.1. For $u, v \in C^{\infty}(\mathscr{U}), \phi \in \mathscr{H}_{\boldsymbol{r}}(\mathscr{U})$, we have the inequality:

$$
\begin{aligned}
& \left|\int_{\mathscr{U}} u(W) v(W) \phi(W) d \mu_{r}(W)\right| \\
& \leqq C\|\phi\|\|v\|_{0} \sum_{0 \leqq j, k \leqq 1} r^{-(j+k) / 2}\left\{\int_{\mathscr{U}}\left|u_{j k}(w)\right|^{2} d \mu_{r}(W)\right\}^{1 / 2} .
\end{aligned}
$$

Proof. To prove this lemma, we view $d \mu_{r}$ as a positive measure on the space of functions of the form $\overline{f f}$, where $f \in C^{\infty}(\mathscr{U})$ such that $\partial f / \partial \bar{\theta}=0$. We write

$$
\begin{aligned}
\left|\int_{\mathscr{U}} u(W) v(W) \phi(W) d \mu_{r}(W)\right| & \leqq \sum_{j, k, l, m}\left|\int_{\mathscr{U}} u_{j k}(w) \bar{\eta}^{j} \eta^{k} v_{l m} \bar{\eta}^{-l} \eta^{m} \phi(W) d \mu_{r}(W)\right| \\
& =\sum_{j, k, l, m}\left|\left(\bar{u}_{j k} \eta^{j} \eta^{l}, v_{l m} \eta^{k} \eta^{m} \phi\right)\right| .
\end{aligned}
$$

We apply the Schwarz inequality to obtain

$$
\left|\int_{\mathscr{U}} u(W) v(W) \phi(W) d \mu_{r}(W)\right| \leqq \sum_{j, k, l, m}\left\|v_{l m}\right\|_{0}\left\|\bar{u}_{j k} \eta^{j} \eta^{l}\right\|\left\|\eta^{k} \eta^{m} \phi\right\| .
$$

The lemma then follows from Proposition III.3.

Applying Lemma V.1 to both $W$ and $Z$ integrations in (V.31), together with the bound (V.33), we obtain

$$
\begin{aligned}
|R| \leqq & C\|g\|_{t}\|f\|_{t}\|\phi\|\|\psi\|\left|\int_{K}(1-z \bar{z})^{-2} d^{2} z\right|^{1 / 2} \\
& \times \sum_{j, k} r^{-(j+k) / 2}\left[\int_{\mathscr{U}} \frac{|w|^{2(4-j-k)}}{\left(1-|w|^{2}\right)^{N}} d \mu_{r}(W)\right]^{1 / 2},
\end{aligned}
$$


for some $t$, where $K$ is a compact set in which $f$ has support. The integral over $K$ is finite and independent of $r$, so we can absorb it into the constant, which we now write $C_{K}$. For the remaining integral over $W$, we have

$$
\begin{aligned}
\int_{\mathscr{U}} \frac{|w|^{2(4-j-k)}}{\left(1-|w|^{2}\right)^{N}} d \mu_{r}(W) & =\frac{r-1}{\pi} \int_{U}|w|^{2(4-j-k)}\left(1-|w|^{2}\right)^{r-2-N} d^{2} w \\
& =\frac{r-1}{\pi} B(4-j-k+1, r-1-N) .
\end{aligned}
$$

For large $r$, we can bound this term by $C r^{-4+j+k}$. Applying this to (V.38), we have

$$
|R| \leqq C_{K} r^{-2}\|g\|_{t}\|f\|_{t}\|\phi\|\|\psi\|
$$

which completes the proof.

\section{Quantization of $\mathbb{C}^{m \mid n}$}

$V I . A$. In this section we describe a quantization of the superspace $\mathbb{C}^{m \mid n}$. The scheme is similar to that of Sect. III, and so some of our arguments will be rather sketchy. Recall that $\mathbb{C}^{m \mid n}$ is the trivial supermanifold $\left(\mathbb{C}^{m}, \mathcal{O}\right)$, where $\mathcal{O}$ is the sheaf of superalgebras on $\mathbb{C}^{m}$ whose space of global sections is $C^{\infty}\left(\mathbb{C}^{m}\right) \otimes \bigwedge\left(\mathbb{C}^{n}\right)$. Let $\theta_{1}, \ldots, \theta_{n}$ denote the standard generators of $\bigwedge\left(\mathbb{C}^{n}\right)$. Then any $f \in C^{\infty}\left(\mathbb{C}^{m \mid n}\right)$ can be written as

$$
f(z, \theta)=\sum_{\alpha, \beta} f_{\alpha \beta}(z) \theta^{\alpha} \bar{\theta}^{\beta}
$$

where the summation runs over all multi-indices $\alpha=\left(\alpha_{1}, \ldots, \alpha_{m}\right), \beta=$ $\left(\beta_{1}, \ldots, \beta_{n}\right), \alpha_{j}, \beta_{k} \in\{0,1\}$, and where $\theta^{\alpha} \bar{\theta}^{\beta}=\theta_{1}^{\alpha_{1}} \ldots \theta_{n}^{\alpha_{n}} \bar{\theta}_{1}^{\beta_{1}} \ldots \bar{\theta}_{n}^{\beta_{n}}$.

In analogy with Sect. II, we say that $f \in C^{\infty}\left(\mathbb{C}^{m \mid n}\right)$ is bounded if the components $f_{\alpha \beta}$ and their derivatives are bounded functions on $\mathbb{C}^{m}$. We let $C_{b}^{\infty}\left(\mathbb{C}^{m \mid n}\right)$ denote the superspace of bounded functions on $\mathbb{C}^{m \mid n}$. We give $C_{b}^{\infty}\left(\mathbb{C}^{m \mid n}\right)$ the topology of a Frechet space by introducing the following family of norms:

$$
\|f\|_{t}:=\sum_{|\mu|+|v| \leqq t} \sum_{\alpha, \beta} \sup \left|\partial_{z}^{\mu} \partial_{\bar{z}}^{v} f_{\alpha \beta}(z)\right|
$$

where $\mu$ and $v$ are multi-indices, $|\mu|:=\sum_{1 \leqq j \leqq m} \mu_{j}$.

A function $f$ is called superholomorphic if $\partial_{\bar{z}_{j}} f=\partial_{\bar{\theta}_{k}} f=0$, or equivalently if

$$
f(z, \theta)=\sum_{\alpha} f_{\alpha}(z) \theta^{\alpha},
$$

with $f_{\alpha}(z)$ holomorphic. Also, we will use the notation $Z:=$ $\left(z_{1}, \ldots, z_{m}, \theta_{1}, \ldots, \theta_{n}\right)$.

The space $\mathbb{C}^{m \mid n}$ has a natural structure of an abelian supergroup. This supergroup acts on $\mathbb{C}^{m \mid n}$ by supertranslations defined by

$$
Z \rightarrow Z^{\prime}=Z+W
$$


On the superalgebra $C^{\infty}\left(\mathbb{C}^{m \mid n}\right)$, one can define a supertranslation invariant super Poisson structure. The two-form

$$
\omega=\sum_{1 \leqq j \leqq m+n}(-1)^{\varepsilon_{j}+1} d \bar{Z}_{j} \wedge d Z_{j}=-Q \bar{Q} Z \bar{Z},
$$

where $Q$ and $\bar{Q}$ are defined in analogy with (II.18), is closed, non-degenerate and supertranslation invariant, and so it is supersymplectic. The associated super Poisson bracket is

$$
\begin{aligned}
\{f, g\}= & -\sum_{1 \leqq j \leqq m}\left(\partial_{z_{j}} f \partial_{\bar{z}_{j}} g-\partial_{\bar{z}_{j}} f \partial_{z_{j}} g\right) \\
& +(-1)^{p(f)+1} \sum_{1 \leqq j \leqq n}\left(\partial_{\bar{\theta}_{j}} f \partial_{\bar{\theta}_{j}} g+\partial_{\bar{\theta}_{j}} f \partial_{\bar{\theta}_{j}} g\right) .
\end{aligned}
$$

VI.B. Let us now consider the following family of Gaussian measures on $\mathbb{C}^{m \mid n}$,

$$
d \mu_{r}(Z)=\frac{r^{m-n}}{\pi^{m}} \exp (-r Z \bar{Z}) d^{2 m} z d^{2 n} \theta .
$$

Proposition VI.1. The form (VI.6) has the following properties

$$
\begin{aligned}
\int_{\mathbb{C}^{m \mid n}} d \mu_{r}(Z) & =1, \\
d \mu_{r}(Z+W) & =\exp (-r Z \bar{W}-r W \bar{Z}-r W \bar{W}) d \mu_{r}(Z) .
\end{aligned}
$$

Proof. The statements follow by a direct calculation.

For $f, g \in C_{b}^{\infty}\left(\mathbb{C}^{m \mid n}\right)$, we define

$$
(f, g)_{r}:=\int_{\mathbb{C}^{m \mid n}} \overline{f(Z)} g(Z) d \mu_{r}(Z) .
$$

As before, when restricted to superholomorphic functions, $(f, g)_{r}$ defines an inner product. We denote the resulting Hilbert space by $\mathscr{H}_{r}\left(\mathbb{C}^{m \mid n}\right)$.

Using the methods explained in Sect. III, we easily establish the following technical fact.

Proposition VI.2. Let $\phi, \psi \in \mathscr{H}_{r}\left(\mathbb{C}^{m \mid n}\right)$. Then, for $f \in C_{b}^{\infty}\left(\mathbb{C}^{m \mid n}\right)$,

$$
\left|\int_{\mathbb{C}^{m \mid n}} \overline{\psi(Z)} f(Z) \phi(Z) d \mu_{r}(Z)\right| \leqq \sum_{\alpha, \beta} r^{-(|\alpha|+|\beta|) / 2}\left\|f_{\alpha \beta}\right\|_{0}\|\psi\|_{r}\|\phi\|_{r} .
$$

In particular,

$$
\left|\int_{\mathbb{C}^{m \mid n}} \overline{\psi(Z)} f(Z) \phi(Z) d \mu_{r}(Z)\right| \leqq\|f\|_{0}\|\psi\|_{r}\|\phi\|_{r} .
$$

The space $\mathscr{H}_{r}\left(\mathbb{C}^{m \mid n}\right)$ carries a natural projective unitary representation of the supergroup of supertranslations, $W \rightarrow U(W)$, where

$$
U(W) \phi(Z):=\exp (r Z \bar{W}-r W \bar{W} / 2) \phi(Z-W) .
$$

Indeed, we verify easily that

$$
U(W+Y)=\sigma(W, Y) U(W) U(Y),
$$


where the cocycle $\sigma(W, Y)$ is defined by

$$
\sigma(W, Y):=\exp \frac{r}{2}(W \bar{Y}-Y \bar{W}) .
$$

VI.C. We set

$$
K^{r}(Z, W):=\exp (r Z \bar{W}),
$$

and make the following proposition.

Proposition VI.3. $K^{r}(Z, W)$ is the Bergman kernel for $\mathscr{H}_{r}\left(\mathbb{C}^{m \mid n}\right)$. Furthermore,

$$
K^{r}(Z+Y, W+Y)=\exp r(Z \bar{Y}+Y \bar{W}) K^{r}(Z, W) \text {. }
$$

Proof. The sequence $\left\{\phi_{\mu, \alpha}\right\}, \mu=\left(\mu_{1}, \ldots, \mu_{m}\right), \mu_{j}=0,1,2, \ldots, \alpha=\left(\alpha_{1}, \ldots, \alpha_{n}\right)$, $\alpha_{j}=1,2$, defined by

$$
\phi_{\mu, \alpha}(Z):=\frac{r^{(|\mu|+|\alpha|) / 2}}{\sqrt{\mu !}} z^{\mu} \theta^{\alpha},
$$

where $\mu !=\mu_{1} ! \ldots \mu_{m} !$, is an orthonormal basis for $\mathscr{H}_{\boldsymbol{r}}\left(\mathbb{C}^{m \mid n}\right)$, and so

$$
\begin{aligned}
\sum_{\mu, \alpha} \phi_{\mu, \alpha}(Z) \overline{\phi_{\mu, \alpha}(W)} & =\sum_{\alpha}\left\{\sum_{\mu} \frac{r^{|\mu|}}{\mu !} z^{\mu} \bar{w}^{\mu}\right\} r^{|\alpha|} \theta^{\alpha} \bar{\theta}^{\alpha} \\
& =\exp (r z \bar{w}) \exp (r \theta \bar{\eta})=\exp (r Z \bar{W}) .
\end{aligned}
$$

VI.D. For $f \in C_{b}^{\infty}\left(\mathbb{C}^{m \mid n}\right)$ and $\phi \in \mathscr{H}_{\boldsymbol{r}}\left(\mathbb{C}^{m \mid n}\right)$, we define the corresponding super Toeplitz operator:

$$
\left(T_{r}(f) \phi\right)(Z):=\int_{\mathbb{C}^{m \mid n}} K^{r}(Z, W) f(W) \phi(W) d \mu_{r}(W) .
$$

As an immediate consequence of Proposition VI.2 we obtain the following estimate.

Proposition VI.4. $T_{r}(f)$ is a bounded operator on $\mathscr{H}_{\boldsymbol{r}}\left(\mathbb{C}^{m \mid n}\right)$. Furthermore,

$$
\left\|T_{r}(f)\right\| \leqq \sum_{\alpha, \beta} r^{-(|\alpha|+|\beta|) / 2}\left\|f_{\alpha \beta}\right\|_{0} .
$$

The algebra $\mathscr{T}_{r}^{\infty}\left(\mathbb{C}^{m \mid n}\right)$ generated by all super Toeplitz operators is a Lie superalgebra whose generators $\sigma_{j}:=T_{r}\left(z_{j}\right), \chi_{k}:=T_{r}\left(\theta_{k}\right), \bar{\sigma}_{j}:=T_{r}\left(\bar{z}_{j}\right), \bar{\chi}_{k}:=T_{r}\left(\bar{\theta}_{k}\right)$, $j=1, \ldots, m, k=1, \ldots, n$, satisfy the following relations,

$$
\begin{aligned}
& {\left[\bar{\sigma}_{j}, \sigma_{k}\right]=r^{-1} \delta_{j k},} \\
& {\left[\sigma_{j}, \bar{\chi}_{k}\right]=0,} \\
& {\left[\chi_{j}, \bar{\chi}_{k}\right]=r^{-1} \delta_{j k},} \\
& {\left[\sigma_{j}, \chi_{k}\right]=0,} \\
& {\left[\chi_{j}, \chi_{k}\right]=0,}
\end{aligned}
$$


and their hermitian conjugates. Strictly speaking, the operators $\sigma$ and $\bar{\sigma}$ are not elements of the algebra $\mathscr{T}_{r}^{\infty}\left(\mathbb{C}^{m \mid n}\right)$, as they are unbounded. The algebra $\mathscr{T}_{r}^{\infty}\left(\mathbb{C}^{m \mid n}\right)$ is generated by $\chi, \bar{\chi}$ and certain bounded functions of $\sigma$ and $\bar{\sigma}$, but we will not elaborate on this point here.

VI.E. We now formulate the main result of this section, namely that the map $C_{b}^{\infty}\left(\mathbb{C}^{m \mid n}\right) \rightarrow \mathscr{T}_{r}^{\infty}\left(\mathbb{C}^{m \mid n}\right)$ given by $T_{r}$ is a deformation quantization. In the next section, we will prove the following two theorems.

Theorem VI.5. For $f \in C_{b}^{\infty}\left(\mathbb{C}^{m \mid n}\right)$, we have

$$
\lim _{r \rightarrow \infty}\left\|T_{r}(f)\right\|_{r}=\left\|f_{00}\right\|_{0} .
$$

Theorem VI.6. For $f, g \in C_{b}^{\infty}\left(\mathbb{C}^{m \mid n}\right)$, such that the components $f_{\alpha \beta}$ are compactly supported, there is a constant $C=C(f, g)$ (depending on $f$ and $g$ ), such that

$$
\left\|T_{r}(f) T_{r}(g)-T_{r}(f g)-r^{-1} \sum_{j}(-1)^{p\left(Z_{j}\right) p(f)+1} T_{r}\left(\partial_{j} f \bar{\partial}_{j} g\right)\right\|_{r} \leqq C r^{-2},
$$

for $r$ sufficiently large.

We can now conclude that $\mathscr{T}_{r}^{\infty}\left(\mathbb{C}^{m \mid n}\right)$ is a quantum deformation of the Poisson superalgebra $C_{b}^{\infty}\left(\mathbb{C}^{m \mid n}\right)$, with the ratio $r^{-1}$ playing the role of Planck's constant.

Theorem VI.7. Under the assumptions of Theorem VI.6,

$$
\left\|r\left[T_{r}(f), T_{r}(g)\right]-T_{r}(\{f, g\})\right\|_{r} \leqq C r^{-1}
$$

for $r$ sufficiently large.

\section{Proof of Deformation Estimates for $\mathbb{C}^{m \mid n}$}

VII.A. Proof of Theorem VI.5. We follow the steps of the proof of Theorem III.9. From Proposition VI.4, we obtain the analog of (IV.1). To prove (IV.2), we use the decomposition (IV.3) with $\phi_{0,0}=1$ and $\gamma_{Z}(W)$ replaced by $W+Z$. The estimates are straightforward, and we leave the details to the reader.

VII.B. Proof of Theorem VI.6. As before, the starting point will be to expand

$$
\left(\phi, T_{r}(f) T_{r}(g) \psi\right)=\int_{\mathbb{C}^{m \mid n} \times \mathbb{C}^{m \mid n}} \overline{\phi(Z)} f(Z) K^{r}(Z, X) g(X) \psi(X) d \mu_{r}(Z) d \mu_{r}(X),
$$

where $\psi, \phi \in \mathscr{H}_{\mathbf{r}}\left(\mathbb{C}^{m \mid n}\right)$, in a power series in $r$. We make the substitution $X=W+Z$, and use the transformation properties of the Bergman kernel to rewrite (VII.1) as

$$
\begin{aligned}
\left(\phi, T_{r}(f) T_{r}(g) \psi\right)= & \int_{\mathbb{C}^{m \mid n} \times \mathbb{C}^{m \mid n}} \overline{\phi(Z)} f(Z) \exp \{-r W \bar{Z}\} \\
& \times g(W+Z) \psi(W+Z) d \mu_{r}(Z) d \mu_{r}(W) .
\end{aligned}
$$


The next step will be to expand $g(W+Z)$ in a Taylor series. We will expand out to order $M:=\max (4,4+m-n)$, as follows (see [9] for the purely bosonic case $n=0$ ):

$$
\begin{aligned}
g(W+Z)= & g(Z)+\sum_{j}\left(W_{j} \partial_{j} g(Z)+\bar{W}_{j} \bar{\partial}_{j} g(Z)\right) \\
& +\frac{1}{2} \sum_{j, k}\left(W_{j} W_{k} \partial_{k} \partial_{j} g(Z)+2 \bar{W}_{j} W_{k} \partial_{k} \bar{\partial}_{j} g(Z)+\bar{W}_{j} \bar{W}_{k} \bar{\partial}_{k} \bar{\partial}_{j} g(Z)\right) \\
& + \text { terms of order } 3 \text { through } M-1+G(Z, W)
\end{aligned}
$$

where the $M^{\text {th }}$ order remainder term is given by

$$
G(Z, W)=\frac{1}{(M-1) !} \int_{0}^{1} d s(1-s)^{M-1} \frac{d^{M}}{d s^{M}} g(s W+Z)
$$

Denote by $I_{p, q}$ the contribution to the integral from the term in the expansion of $g$ with $p W$ 's and $q \bar{W}$ 's, and let $R$ denote the contribution of the remainder term. In evaluating these terms, we will make use of the following facts. For a holomorphic function $h$ on $\mathbb{C}^{m \mid n}$ and a multi-index $\mu$,

$$
\int_{\mathbb{C}^{m \mid n}} \bar{w}^{\mu} h(W) d \mu_{r}(W)=\frac{1}{\mu !} \partial_{z}^{\mu} h(0) \int_{\mathbb{C}^{m \mid n}} \prod_{1 \leqq j \leqq m}\left|w_{j}\right|^{2 \mu_{j}} d \mu_{r}(W),
$$

and

$$
\int_{\mathbb{C}^{m \mid n}} \bar{w}^{\mu} \bar{\eta}_{\alpha} h(W) d \mu_{r}(W)=\frac{1}{\mu !} \partial_{\theta_{\alpha}} \partial_{z}^{\mu} h(0) \int_{\mathbb{C}^{m \mid n}} \prod_{1 \leqq j \leqq m}\left|w_{j}\right|^{2 \mu_{j}} \bar{\eta}_{\alpha} \eta_{\alpha} d \mu_{r}(W) .
$$

The lowest order term in the expansion is

$$
I_{0,0}=\int_{\mathbb{C}^{m \mid n} \times \mathbb{C}^{m \mid n}} \overline{\phi(Z)} f(Z) \exp \{-r W \bar{Z}\} g(Z) \psi(W+Z) d \mu_{r}(Z) d \mu_{r}(W) .
$$

The integrand is holomorphic in $W$, so we apply (VII.5) to get

$$
I_{0,0}=\int_{\mathbb{C}^{m \mid n} \times \mathbb{C}^{m \mid n}} \overline{\phi(Z)} f(Z) g(Z) \psi(Z) d \mu_{r}(Z)=\left(\phi, T_{r}(f g) \psi\right),
$$

which gives the first term in the expansion of $T_{r}(f) T_{r}(g)$ in Theorem VI.6. In the same fashion we also easily show that $I_{p, q}=0$, for $p>q$.

The next non-zero term is thus $I_{0,1}$, which is given by

$$
I_{0,1}=\sum_{j} \int_{\mathbb{C}^{m \mid n} \times \mathbb{C}^{m \mid n}} \bar{\phi}(Z) f(Z) \exp \{-r W \bar{Z}\} \bar{W}_{j} \bar{\partial}_{j} g(Z) \psi(W+Z) d \mu_{r}(Z) d \mu_{r}(W) .
$$

We now use (VII.5) and (VII.6) as well as the integrals

$$
\int_{\mathbb{C}^{m \mid n}}\left|w_{\alpha}\right|^{2} d \mu_{r}(W)=\int_{\mathbb{C}^{m \mid n}} \bar{\eta}_{\alpha} \eta_{\alpha} d \mu_{r}(W)=\frac{1}{r},
$$


to obtain

$$
\begin{aligned}
I_{0,1}=\frac{1}{r} & \sum_{j}(-1)^{\varepsilon_{j}(p(g)+1)} \int_{\mathbb{C}^{m \mid n}} \overline{\phi(Z)} f(Z) \bar{\partial}_{j} g(Z) \exp (r Z \bar{Z}) \\
\times & {\left[\frac{\partial}{\partial W_{j}} \psi(W+Z) \exp \{-r(W+Z) \bar{Z}\}\right]_{W=0} d \mu_{r}(Z), }
\end{aligned}
$$

where $\varepsilon_{j}:=p\left(Z_{j}\right)$. Applying the chain rule gives

$$
\begin{aligned}
I_{0,1}= & \frac{1}{r} \sum_{j}(-1)^{\varepsilon_{j}(p(g)+1)} \int_{\mathbb{C}^{m \mid n}} \overline{\phi(Z)} f(Z) \bar{\partial}_{j} g(Z) \\
& \times \partial_{j}\{\psi(Z) \exp (-r Z \bar{Z})\} \exp \{r Z \bar{Z}\} d \mu_{r}(Z) .
\end{aligned}
$$

Integrating by parts we obtain

$$
\begin{aligned}
I_{0,1}= & -\frac{1}{r} \sum_{j}(-1)^{\varepsilon_{j} p(f)} \int_{\mathbb{C}^{m \mid n}} \overline{\phi(Z)} \partial_{j} f(Z) \bar{\partial}_{j} g(Z) \psi(Z) d \mu_{r}(Z) \\
& -\frac{1}{r} \sum_{j}(-1)^{p(g) \varepsilon_{j}} \int_{\mathbb{C}^{m \mid n}} \overline{\phi(Z)} f(Z) \partial_{j} \bar{\partial}_{j} g(Z) \psi(Z) d \mu_{r}(Z) .
\end{aligned}
$$

Now consider the term $I_{1,1}$, which is given by

$$
\begin{aligned}
I_{1,1}= & \sum_{j, k} \int_{\mathbb{C}^{m \mid n} \times \mathbb{C}^{m \mid n}} \overline{\phi(Z)} f(Z) \exp \{-r W \bar{Z}\} \bar{W}_{j} W_{k} \partial_{k} \bar{\partial}_{j} g(Z) \\
& \times \psi(W+Z) d \mu_{r}(Z) d \mu_{r}(W) .
\end{aligned}
$$

Using (VII.5) and (VII.6), together with (VII.10), we can perform the $W$ integration to get

$$
I_{1,1}=\frac{1}{r} \sum_{j}(-1)^{p(g) \varepsilon_{j}} \int_{\mathbb{C}^{m \mid n}} \overline{\phi(Z)} f(Z) \partial_{j} \bar{\partial}_{j} g(Z) \psi(Z) d \mu_{r}(Z) .
$$

This exactly cancels the second term in (VII.13), so that we obtain

$$
I_{0,1}+I_{1,1}=-\frac{1}{r} \sum_{j}(-1)^{\varepsilon_{j} p(f)}\left(\phi, T_{r}\left(\partial_{j} f \bar{\partial}_{j} g\right) \psi\right),
$$

as desired. Of the remaining second order terms, $I_{2,0}=0$, and $I_{0,2}$ is given by

$$
\begin{aligned}
I_{0,2}= & \frac{1}{2} \sum_{j, k} \int_{\mathbb{C}^{m \mid n} \times \mathbb{C}^{m \mid n}} \overline{\phi(Z)} f(Z) \exp (-r W \bar{Z}) \bar{W}_{j} \bar{W}_{k} \bar{\partial}_{k} \bar{\partial}_{j} g(Z) \\
& \times \psi(W+Z) d \mu_{r}(Z) d \mu_{r}(W) .
\end{aligned}
$$

We want to bound this term for large $r$. First, we use (VII.5) and (VII.6) as before, to evaluate the $W$ integration. Then observe that for any $N \geqq 0$,

$$
\int_{\mathbb{C}^{m \mid n}} \prod_{1 \leqq j \leqq N} W_{k_{j}} \bar{W}_{k_{j}} d \mu_{r}(W)=\kappa_{N}\left(k_{1}, \ldots, k_{N}\right) r^{-N},
$$


with $\kappa_{N}\left(k_{1}, \ldots, k_{N}\right)$ independent of $r$, and so we can make the bound

$$
\begin{aligned}
\left|I_{0,2}\right| \leqq & C r^{-2} \mid \sum_{j, k} \int_{\mathbb{C}^{m \mid n}} \overline{\phi(Z)} f(Z) \bar{\partial}_{k} \bar{\partial}_{j} g(Z) \exp (r Z \bar{Z}) \\
& \times\left[\frac{\partial^{2}}{\partial W_{j} \partial W_{k}} \psi(W+Z) \exp \{-r(W+Z) \bar{Z}\}\right]_{W=0} d \mu_{r}(Z) \mid
\end{aligned}
$$

As before, we apply the chain rule to convert $W$ derivatives into $Z$ derivatives, and then integrate by parts to move these derivatives onto $f$ and $g$. Using Proposition VI.2, we can extract the sup norms of the derivatives of $f$ and $g$ from the integral. In this way, we obtain the bound

$$
\left|I_{0,2}\right| \leqq C r^{-2}\|f\|_{t}\|g\|_{t}\|\psi\|\|\phi\|
$$

for some $t$.

We can apply the same arguments used for $I_{0,2}$ to bound the explicit terms of order three through $M-1$ in (VII.3). The result is that

$$
\left|I_{p, q}\right| \leqq C r^{-2}\|f\|_{t}\|g\|_{t}\|\psi\|\|\phi\|
$$

for $3 \leqq p+q \leqq M-1$.

Now we turn to the remainder term, which is equal to

$$
R=\int_{\mathbb{C}^{m \mid n} \times \mathbb{C}^{m \mid n}} \overline{\phi(Z)} f(Z) G(Z, W) \psi(W+Z) \exp (-r W \bar{Z}) d \mu_{r}(Z) d \mu_{r}(W) .
$$

Note that

$$
\psi(W+Z) \exp (-r W \bar{Z})=\exp (r Z \bar{Z} / 2)(U(-Z) \psi)(W)
$$

where $U$ is defined by (VI.11). Denote $U(-Z) \psi(W)$ by $\psi_{Z}(W)$, and observe that

$$
\left\|\psi_{Z}\right\|=\|\psi\|
$$

The remainder term can now be written in the form

$$
R=\int_{\mathbb{C}^{m \mid n} \times \mathbb{C}^{m \mid n}} \overline{\phi(Z)} f(Z) G(Z, W) \psi_{Z}(W) \exp (r Z \bar{Z} / 2) d \mu_{r}(Z) d \mu_{r}(W)
$$

Lemma VII.1. For $u, v \in C^{\infty}\left(\mathbb{C}^{m \mid n}\right), \phi \in \mathscr{H}_{r}\left(\mathbb{C}^{m \mid n}\right)$, we have

$$
\begin{aligned}
& \left|\int_{\mathbb{C}^{m \mid n}} u(w) v(w) \phi(w) d \mu_{r}(W)\right| \\
& \leqq C\|\phi\|\|v\|_{0} \sum_{\alpha, \beta} r^{-(|\alpha|+|\beta|) / 2}\left\{\int_{\mathbb{C}^{m \mid n}}\left|u_{\alpha \beta}(w)\right|^{2} d \mu_{r}(W)\right\}^{1 / 2} .
\end{aligned}
$$

Proof. The proof follows closely that of Lemma V.1. 
We write the function $G(Z, W)$ as a sum of terms of the form $G^{\mu v}(Z, W) \bar{\eta}^{\mu} \eta^{v}$. We use Lemma VII.1 to obtain

$$
\begin{aligned}
& \left|\int_{\mathbb{C}^{m \mid n} \times \mathbb{C}^{m \mid n}} \overline{\phi(Z)} f(Z) G^{\mu v}(Z, W) \bar{\eta}^{\mu} \eta^{v} \exp (r Z \bar{Z} / 2) \psi_{Z}(W) d \mu_{r}(Z) d \mu_{r}(W)\right| \\
& \leqq C_{K} r^{(m-n) / 2}\|f\|_{t}\|g\|_{t}\|\phi\|\|\psi\| \sum_{\mu, v} r^{-(|\mu|+|v|) / 2}\left\{\int_{\mathbb{C}^{m \mid n}}|w|^{2(M-|\mu|-|v|)} d \mu_{r}(W)\right\}^{1 / 2} \\
& \leqq C_{K} r^{-(M-m+n) / 2}\|f\|_{t}\|g\|_{t}\|\phi\|\|\psi\|,
\end{aligned}
$$

which completes the proof, since $M-m+n \geqq 4$.

Acknowledgement. We would like to thank Lewis Coburn for very helpful comments on the manuscript. We also wish to thank the anonymous referee for a constructive remark.

\section{References}

1. Axler, S., Conway, J.B., McDonald, G.: Toeplitz operators on Bergman spaces. Can. J. Math. 34, 466-483 (1982)

2. Bayen, R., Flato, M., Fronsdal, C., Lichnerowicz, A., Sternheimer, D.: Deformation theory and quantization. Ann. Phys. 110, 61-151 (1978)

3. Berezin, F.A.: Quantization. Math. USSR Izvestija 8, 1109-1165 (1974)

4. Berezin, F.A.: Quantization in complex symmetric spaces. Math. USSR Izvestija 9, 341-379 (1975)

5. Berezin, F.A.: General concept of quantization. Commun. Math. Phys. 40, 153-174 (1975)

6. Berezin, F.A.: Models of Gross-Neveu type are quantization of a classical mechanics with nonlinear phase space. Commun. Math. Phys. 63, 131-153 (1978)

7. Berezin, F.A.: Introduction to Superanalysis. Dordrecht: D. Reidel 1987

8. Borthwick, D., Lesniewski, A., Upmeier, H.: Non-perturbative deformation quantization of Cartan domains. J. Funct. Anal., to appear

9. Coburn, L.A.: Deformation estimates for the Berezin-Toeplitz quantization. Commun. Math. Phys. 149, 415-424 (1992)

10. Connes, A.: Non-commutative differential geometry. Publ. Math. IHES 62, 94-144 (1986)

11. Douglas, R.: Banach Algebra Techniques in Operator Theory. New York, London: Academic Press 1972

12. Klimek, S., Lesniewski, A.: Quantum Riemann surfaces, I. The unit disc. Commun. Math. Phys. 146, 103-122 (1992)

13. Klimek, S., Lesniewski, A.: Quantum Riemann surfaces, II. The discrete series. Lett. Math. Phys. 24, 125-139 (1992)

14. Klimek, S., Lesniewski, A.: A two parameter deformation of the unit disc. J. Funct. Anal., to appear

15. Kostant, B.: Graded manifolds, graded Lie theory and prequantization. Lect. Notes in Math. 570. Berlin, Heidelberg, New York: Springer 1977

16. Kostant, B., Sternberg, S.: Symplectic reduction, BRS cohomology, and infinite-dimensional Clifford algebras. Ann. Phys. 176, 49-113 (1987)

17. Manin, Yu.: Gauge Field Theory and Complex Geometry. Berlin Heidelberg New York: Springer 1988

18. Rieffel, M.: Deformation quantization of Heisenberg manifolds. Commun. Math. Phys. 122, 531-562 (1989)

19. Rieffel, M.: Deformation quantization for actions of $\mathbb{R}^{d}$. Preprint 1991

20. Sheu, A.J.-L., Lu, J.-H., Weinstein, A.: Quantization of the Poisson SU(2) and its Poisson homogeneous space - the 2-sphere. Commun. Math. Phys. 135, 217-232 (1991)

21. Upmeier, H.: Toeplitz $\mathbb{C}^{*}$-algebras on bounded symmetric domains. Ann. Math. 119, 549-576 (1984) 